\title{
Parametric study of EEG sensitivity to phase noise during face
} processing

\author{
Guillaume A Rousselet*1, Cyril R Pernet ${ }^{2}$, Patrick J Bennett ${ }^{3}$ and \\ Allison B Sekuler ${ }^{3}$
}

Address: ${ }^{1}$ Centre for Cognitive Neuroimaging (CCNi) and Department of Psychology, University of Glasgow, Glasgow, UK, ${ }^{2}$ SFC Brain Imaging Research Centre, Division of Clinical Neurosciences, Western General Hospital, Edinburgh, UK and ${ }^{3}$ McMaster University, Department of Psychology, Behaviour \& Neuroscience, Hamilton, ON, Canada

Email: Guillaume A Rousselet* - g.rousselet@psy.gla.ac.uk; Cyril R Pernet - cyril.pernet@ed.ac.uk; Patrick J Bennett - bennett@mcmaster.ca; Allison B Sekuler - sekuler@mcmaster.ca

* Corresponding author

Published: 3 October 2008

BMC Neuroscience 2008, 9:98 doi:10.1 |86//47|-2202-9-98
Received: 24 June 2008

Accepted: 3 October 2008

This article is available from: http://www.biomedcentral.com/I47I-2202/9/98

(c) 2008 Rousselet et al; licensee BioMed Central Ltd.

This is an Open Access article distributed under the terms of the Creative Commons Attribution License (http://creativecommons.org/licenses/by/2.0), which permits unrestricted use, distribution, and reproduction in any medium, provided the original work is properly cited.

\begin{abstract}
Background: The present paper examines the visual processing speed of complex objects, here faces, by mapping the relationship between object physical properties and single-trial brain responses. Measuring visual processing speed is challenging because uncontrolled physical differences that co-vary with object categories might affect brain measurements, thus biasing our speed estimates. Recently, we demonstrated that early event-related potential (ERP) differences between faces and objects are preserved even when images differ only in phase information, and amplitude spectra are equated across image categories. Here, we use a parametric design to study how early ERP to faces are shaped by phase information. Subjects performed a two-alternative force choice discrimination between two faces (Experiment I) or textures (two control experiments). All stimuli had the same amplitude spectrum and were presented at II phase noise levels, varying from $0 \%$ to $100 \%$ in $10 \%$ increments, using a linear phase interpolation technique. Single-trial ERP data from each subject were analysed using a multiple linear regression model.
\end{abstract}

Results: Our results show that sensitivity to phase noise in faces emerges progressively in a short time window between the PI and the NI70 ERP visual components. The sensitivity to phase noise starts at about 120-130 ms after stimulus onset and continues for another 25-40 ms. This result was robust both within and across subjects. A control experiment using pink noise textures, which had the same second-order statistics as the faces used in Experiment I, demonstrated that the sensitivity to phase noise observed for faces cannot be explained by the presence of global image structure alone. A second control experiment used wavelet textures that were matched to the face stimuli in terms of second- and higher-order image statistics. Results from this experiment suggest that higher-order statistics of faces are necessary but not sufficient to obtain the sensitivity to phase noise function observed in response to faces.

Conclusion: Our results constitute the first quantitative assessment of the time course of phase information processing by the human visual brain. We interpret our results in a framework that focuses on image statistics and single-trial analyses. 


\section{Background}

In primates, visual object processing unfolds from the retina to higher-order cortical areas through a hierarchy of processing steps. Although, at the neuronal level, lateral and feedback connections are integrated to the feedforward sweep of information [1,2], at the functional level, neuronal mechanisms can still be conceptualized as performing rapid transformations of the input retinal activation to achieve increasingly refined representations [3]. A fundamental question in vision science is thus how to uncover the mechanisms by which the pattern of retinal activation is progressively transformed into a code that is useful for making behavioural decisions. In recent years there has been an on-going debate as to what stimuli are best for probing visual neuronal mechanisms. This debate stems mostly from the study of neurons in V1, the primary visual cortex, and whether their visual response properties can be better understood by using simple well-controlled stimuli [4], or natural scene stimuli, the type of stimuli the visual system might have evolved to apprehend best [5]. At the other end of the visual cortical hierarchy, in higher order visual areas, no such debate exists since those areas are mostly responsive to complex objects, and not to simple patterns [6-10]. In those areas, the emphasis has been put on object categories and their relative specificity [11]. Although interesting in itself, the category-related parcelling of the visual cortex ignores the question of the transformation mechanisms taking place along the visual hierarchy. Because these processes occur very fast, critical information processing events may be observed at the time-scale of EEG (electroencephalography) [3,12-14]. In humans, EEG (as well as MEG more recently) has revealed a cascade of neuronal activations following stimulus presentation [15]. Within 200 ms, neuronal activity has been reported that dissociates among various object categories, in particular faces and words [16-19]. In particular, the larger ERP component to faces and words, compared to other control categories, and peaking at about $170 \mathrm{~ms}$, the N170 [20-23], has been the subject of much debate about its categorical sensitivity [24]. Early activity, in the time window of the P1 component ( $80-120 \mathrm{~ms}$ ), also has been discussed as a potential marker of complex object processing [25-27].

On-going controversies about the time-course of object processing are due, in part, to the difficulty associated with controlling the effects of low-level sensory variables on higher-order perceptual operations. In classic categorical designs that are used to assess object-processing speed, uncontrolled physical properties tend to co-vary with the object categories that are contrasted. Such physical properties might introduce biases in our brain measurements that are unrelated to the higher-level object processing that are meant to be measured, but instead reflect the extraction of visual information by lower levels of the visual hierarchy $[25,28]$.

Recent advances have revealed that the activity in the N170 time window, but not earlier activity, is related to the extraction of task-related information (EEG: [14,2931]; MEG: [32,33]). These advances were made possible by a tight control of the stimulus space that relied on parametric, rather than categorical, designs. Parametric designs are well suited to explore brain dynamics in a systematic fashion because, by varying one or several parameters along a continuum, they can provide a genuine information space and stronger constraints on statistical analyses [14,34,35].

The goal of our project was to build on this new literature using parametric designs, to determine what image properties drive early responses to objects. Images are characterized by their amplitude and phase spectra in the Fourier domain. Differences in amplitude spectra are not likely to explain early responses to faces and objects because equating amplitude spectra across stimuli preserve those differences $[28,30,31,36,37]$. For instance, in Figure 1, the top left textures and the top right faces all have the same amplitude spectrum, but have different phase spectra. Therefore, phase information is the most likely candidate, a conclusion that has been reached previously for psychophysical data [38-41]. When contrasting face stimuli to noise textures created by complete randomization of the phase information while keeping the amplitude information constant, the earliest ERP differences occur at about 120-140 ms after stimulus onset [28,30,31,37,42,43]. Although this might be the time at which object-related global phase information is extracted by the visual system, the precise time course of this process is still unknown, as is the way phase information influences early cortical responses to objects. We addressed these questions by manipulating phase information systematically along a continuum while subjects discriminated between two faces briefly presented on a screen (Figure 1). Mutual phase information, with respect to the target images, was manipulated by adding phase noise in linear steps. Analyses were performed on each subject at the single-trial level, using a linear regression model that contained only predictors related to physical image characteristics, including stimulus type (e.g., face 1 or face 2 ) and percentage of phase information (ranging from 100\%, i.e. original stimuli, to $0 \%$, i.e. equivalent to the phase scrambled textures used in earlier experiments).

Previous experiments have shown that behavioural performance of human observers in natural scene categorisation tasks is very resistant to linear alterations of the phase spectrum by introduction of phase noise $[40,44,45]$. This finding might be explained by the existence of non-linear- 

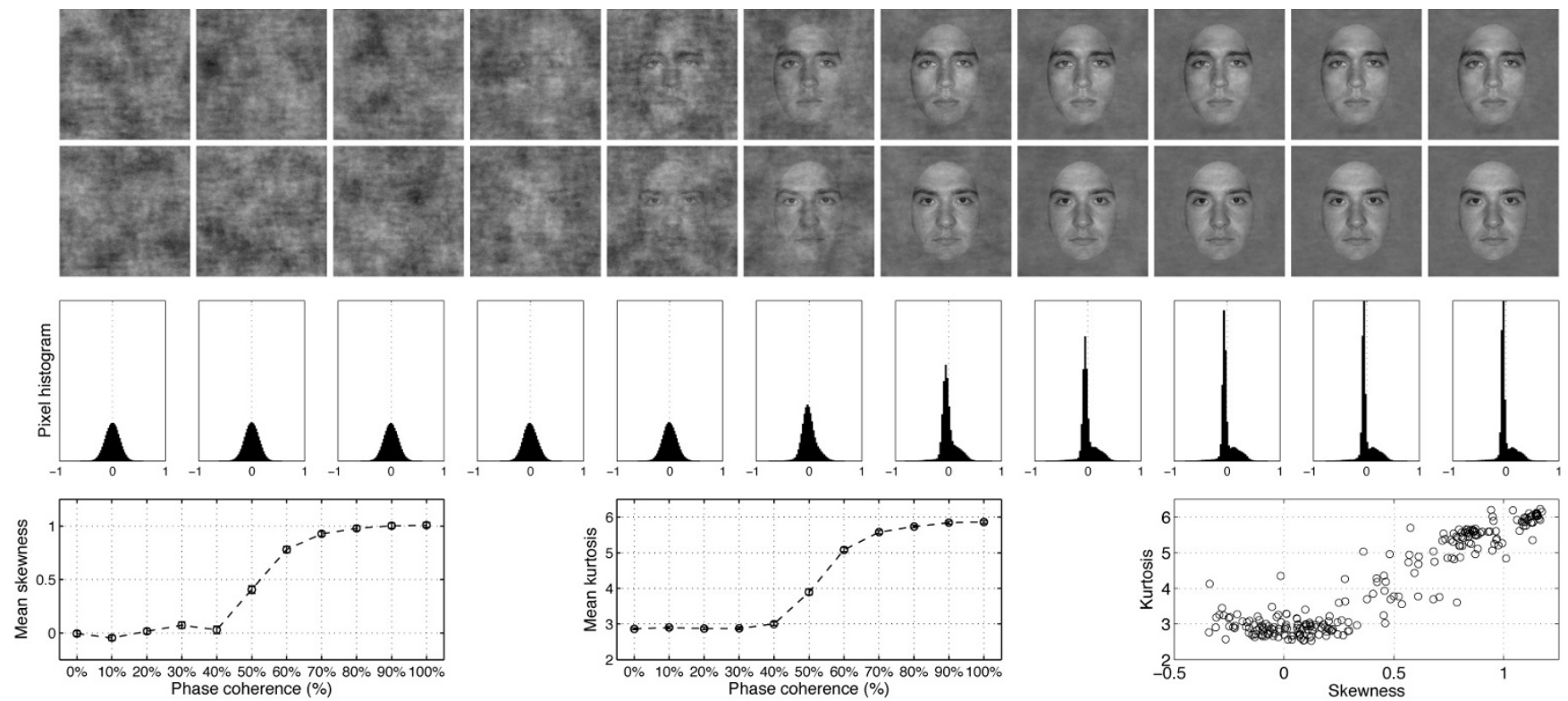

Figure I

Examples of stimuli used in Experiment I. The first two rows show the 22 stimuli presented to one observer during the first block of the experiment. The observer discriminated the same two faces during the whole experiment. The noise level varied from $100 \%$ (left side; $0 \%$ phase coherence) to $0 \%$ (right side; $100 \%$ phase coherence). Note that at each level of phase coherence the structure of the noise that was mixed with the original image was different, so that the task could not be performed based on the spatial characteristics of the noise. Histograms in the third row show the distribution of pixel contrasts averaged across all stimuli seen by this observer at each level of phase coherence. Starting with a Gaussian distribution (left), the pixel histograms become increasingly skewed and kurtotic with increasing phase coherence (right - the $y$-axes on the histograms are all the same). This relationship is depicted in the last row, showing the mean skewness (left), and mean kurtosis (middle), as a function of phase coherence. The error bars correspond to $95 \%$ confidence interval computed using a bootstrap percentile technique ( 1000 resamples). In the bottom right end graph, kurtosis for each image (each circle) is expressed as a function of skewness. Although the two statistical descriptors are correlated, the relationship is not linear. As demonstrated below, EEG amplitude is more sensitive to the kurtosis of the image than its skewness.

ities in higher-order image statistics introduced by linear manipulations of phase information [46]. In particular, phase manipulations affect the skewness and kurtosis of natural images (Figure 1), i.e. their $3^{\text {rd }}$ and $4^{\text {th }}$ order statistics. Skewness is a measure of the asymmetry of a distribution. It equals zero for a Gaussian distribution, and is negative or positive for distributions with more variance to the left or to the right of their centre. Kurtosis is a measure of how peaky and heavy-tailed a distribution is, and equals 3 for a Gaussian distribution [47]. An image with a high kurtosis contains a large proportion of almost identical pixels, and few pixels that differ significantly from the modal value. Skewness and kurtosis are defined as the normalized $3^{\text {rd }}$ and $4^{\text {th }}$ moments about the mean of the pixel luminance distribution $[48,49]$ :

Skewness $=\frac{1}{N}\left(\sum_{i}^{N}\left(x_{i}-\bar{x}\right)^{3}\right) \frac{1}{\left(\sigma^{2}\right)^{3 / 2}} ;$ Kurtosis $=\frac{1}{N}\left(\sum_{i}^{N}\left(x_{i}-\bar{x}\right)^{4}\right) \frac{1}{\left(\sigma^{2}\right)^{2}}$

where $\mathrm{N}$ is the sample size of the distribution, $\mathrm{x}_{\mathrm{i}}$ the value of the $i$-th member of the distribution, $\overline{\mathrm{x}}$ the mean of the $i$ values, and $\sigma^{2}$ the variance (for a good visualisation of skewness and kurtosis in the context of $1 / \mathrm{f}$ wavelet textures, see Figure 1 from [48]). Kurtosis, in particular, seems to be related to the presence of edges and local contours in natural images, and might thus be a better indicator of image structure than global phase coherence per se [46,50-52]. Therefore, we included skewness and kurtosis as predictors in our linear regression model. We report data showing, in response to face stimuli, sensitivity to phase noise that emerged very rapidly at the transition between the P1 and the N170 components, in the 120$150 \mathrm{~ms}$ after stimulus onset time window.

\section{Methods \\ Participants}

A total of 10 subjects participated in one main experiment and two control experiments. Experiment 1 included eight subjects (five males and three females). Four were tested twice (test-retest, on two different days), leading to a total of 12 experimental sessions. Only half of the sub- 
jects were tested twice because of the robust replication of the effects we obtained with the first four subjects (see Results). Subjects' mean age was 24 years old $(\mathrm{min}=21$, $\max =28, S D=2.5$ ); seven were right handed. Experiment 2 included four male subjects, and all of them were tested twice (mean age $27, \min =24, \max =29, \mathrm{SD}=2.4$; three right handed). Four subjects (one female) participated in Experiment 3, one of whom was tested twice (mean age $25, \min =22, \max =29, \mathrm{SD}=3$; three were right handed) . All subjects gave written informed consent and had normal or corrected-to-normal vision. Five subjects participated in one experiment only, four participated in two experiments, and only one subject participated in three experiments. This last subject, RXL, was singled-out for this reason in the results. Among the 10 different individuals, 4 received $\$ 10 /$ hour for their participation; the others were members of the laboratory and were not compensated for participation. The McMaster University Research Ethics Board approved the research protocol.

\section{Stimuli}

One pair of female faces and one pair of male faces were selected from a set of 10 faces used in previous experiments $[36,53,54]$. Each subject saw only two faces, from the same gender, and male and female faces were counterbalanced across subjects. These faces were front-view greyscale photographs cropped within a common oval frame and pasted on a uniform $10^{\circ} \times 10^{\circ}$ background (Figure $1)$. Face stimuli all had the same mean amplitude spectrum and thus differed only in terms of phase information, which carries most of the form information $[55,56]$. We created four noise textures by randomizing the phase of the four faces. Thus, these patterns, which we refer to as pink noise textures, had the same amplitude spectrum as the faces, but differed from faces in terms of higher-order statistics. We also created four wavelet textures, each matched for the global image statistics of one of the faces. These textures not only matched the skewness and kurtosis of the original face stimuli, they also matched properties such as local and long-distance multiscale phase correlations. The textures were created with the Matlab toolbox provided by Portilla and Simoncelli ([57], http:// www.cns.nyu.edu/ lcv/texture/), with the parameters set to four scales, four orientations, a $9 \times 9$ spatial neighbourhood, and 50 iterations.

Phase spectra were manipulated using the weighted mean phase technique (WMP, [46]), so that images were characterized by their percentage of phase coherence. Starting from the original phase of an image $\phi_{\text {image }}$ the final phase $\phi_{\text {final }}$ was computed by the following equation:

$$
\varphi_{\text {final }}= \begin{cases}\tan ^{-1}\left(S_{\varphi} / C_{\varphi}\right) & C_{\varphi}>0 \\ \tan ^{-1}\left(S_{\varphi} / C_{\varphi}\right)+\pi & C_{\varphi}<0, S_{\varphi}>0 \\ \tan ^{-1}\left(S_{\varphi} / C_{\varphi}\right)+2 \pi & C_{\varphi}<0, S_{\varphi}<0\end{cases}
$$

where:

$$
\begin{aligned}
& S_{\varphi}=w \sin \left(\varphi_{\text {image }}\right)+(1-w) \sin \left(\varphi_{\text {noise }}\right) \quad w[0,1] \\
& C_{\varphi}=w \cos \left(\varphi_{\text {image }}\right)+(1-w) \cos \left(\varphi_{\text {noise }}\right)
\end{aligned}
$$

This technique takes into account the directional nature of phase, assuring that phases are uniformly distributed after transformation. In comparison, a strict linear blend would lead to an over-representation of phases around $0^{\circ}$. Thus, WMP has the advantage over a linear blend technique to produce monotonic changes in third-order (skewness) and fourth-order (kurtosis) image statistics, as illustrated at the bottom part of Figure 1 and in [46]. Kurtosis is often used as a measure of image sparseness and is highly correlated with the representation of phase structure, high levels of kurtosis corresponding to local phasecongruent structures such as edges [50].

For all stimuli, pixel contrasts ranged between -1 and 1 , with a mean of 0 . RMS contrast was kept constant across all levels of phase coherence.

\section{Experimental design}

Subjects sat in a dimly lit sound-attenuated booth. Viewing distance was maintained at $90 \mathrm{~cm}$ with a chinrest. Stimuli were presented for about $53 \mathrm{~ms}$ ( 4 frames at 75 $\mathrm{Hz})$ on a Sony Trinitron GDM-F520 monitor $(800 \times 600$ pixels, effective height and width: $40.5 \times 30.5 \mathrm{~cm})$. Subjects were given unlimited time to respond by pressing ' 1 ' or ' 2 ' on the numerical pad of the keyboard to indicate which stimulus had been displayed (Figure 2). In Experiment 1 , subjects had to discriminate between two faces; in Experiment 2 between two pink noise textures; and in Experiment 3 between two wavelet noise textures. In all experiments, subjects were told to emphasize response accuracy, not speed. The button/identity association was assigned randomly for each subject. An experiment consisted of 12 blocks of 132 trials (1584 trials in total with 144 trials per level of phase coherence). Within each block, there were six repetitions of each face or texture in 11 phase coherence levels. Each block was preceded by practice trials that allowed subjects to learn the stimuluskey association (10 in Experiment 1, and 20 in the two control experiments). A regular trial was organized as follows: A blank screen was presented for $1000 \mathrm{~ms}$, followed by a small fixation cross (i.e., a $0.3 \mathrm{deg}$ ' + ' in the middle of the screen) for $200 \mathrm{~ms}$, after which another blank screen was presented for a random duration ranging from 500 to $1000 \mathrm{~ms}$. Then a stimulus was presented for $53 \mathrm{~ms}$, followed by a blank screen that stayed on until subjects pro- 


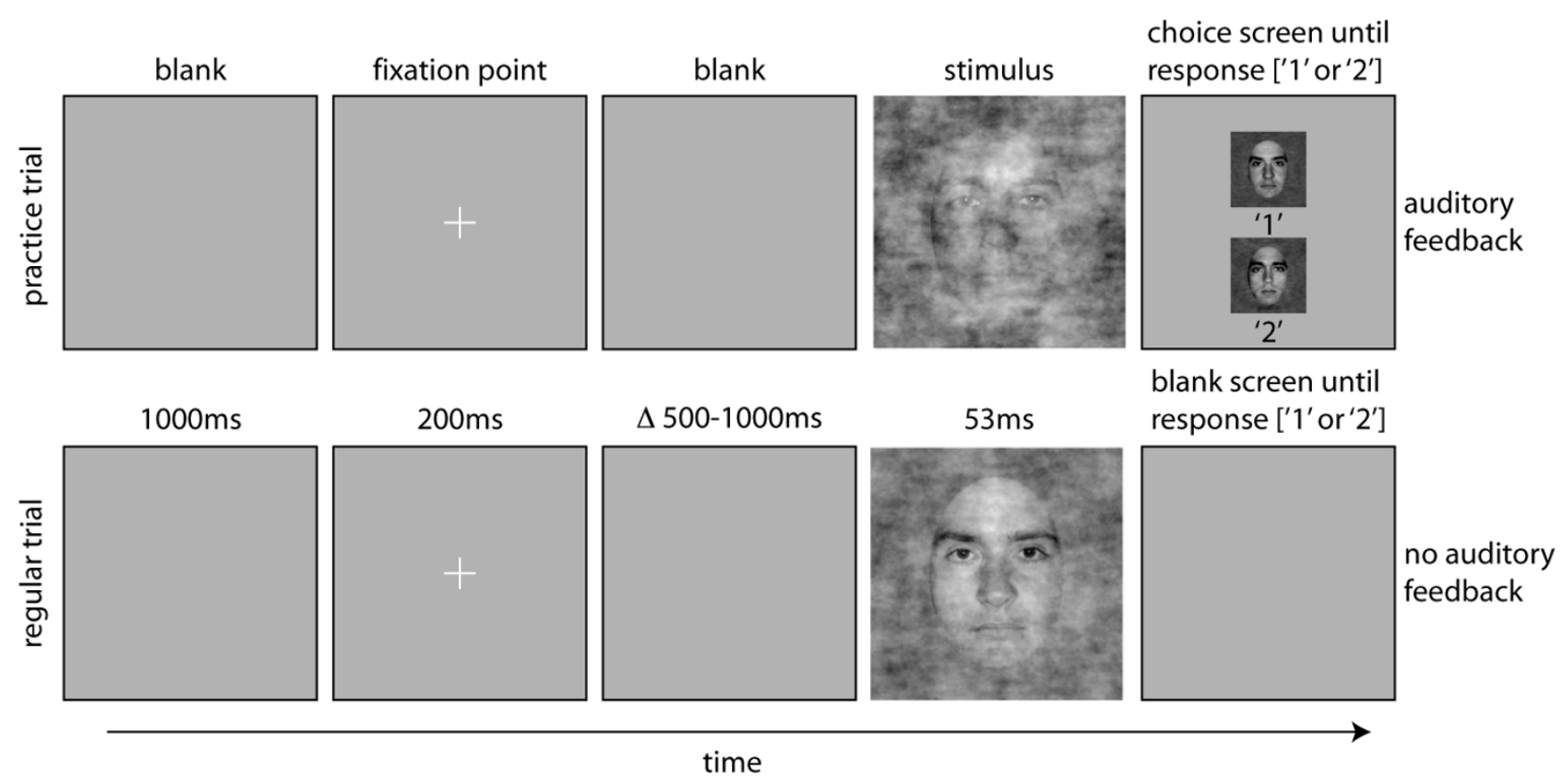

\section{Figure 2}

Organisation of practice trials (top row) and regular experimental trials (bottom row) in all experiments. The stimuli are examples taken from the main experiment that used faces (Experiment I). A trial started with a blank screen for $1000 \mathrm{~ms}$, followed by the presentation of a fixation point for $200 \mathrm{~ms}$. Then, after a random delay ranging from 500 to $1000 \mathrm{~ms}$, a stimulus was presented for $53 \mathrm{~ms}$. During practice trials, a choice screen appeared immediately after the stimulus, showing the two targets of the experiment and their associated response keys. The screen stayed on until the subject's response, which was followed by auditory feedback, before the trial sequence resumed. During regular trials, a blank screen appeared immediately after the stimulus, and remained on until the subject's response. No feedback was provided during regular trials. Note that stimuli are not drawn to scale.

vided their response. Practice trials were very similar, except that immediately after the presentation of the stimulus, a choice screen appeared that showed each face or texture simultaneously, one above the other, with the corresponding label below each item. Auditory feedback was provided after the subject pressed a response key, with low- and high-pitch tones indicating incorrect and correct responses. Feedback was provided only during practice trials.

\section{EEG recording and analysis}

EEG data were acquired with a 256-channel Geodesic Sensor Net (Electrical Geodesics Inc., Eugene, Oregon, [58]). Analog signal was digitized at $500 \mathrm{~Hz}$ and band-pass filtered between $0.1 \mathrm{~Hz}$ and $200 \mathrm{~Hz}$. Impedances were kept below $50 \mathrm{k} \Omega$. Subjects were asked to minimize blinking, head movement, and swallowing. Subjects were then given a description of the task. EEG data were referenced on-line to electrode $\mathrm{Cz}$ and re-referenced off-line to an average reference. The signal was then low-pass filtered at $30 \mathrm{~Hz}$ and bad channels removed, with no interpolation. The $30 \mathrm{~Hz}$ low-pass filter was justified by a previous study in which we showed that the differential activity evoked by faces and objects is contained mostly in a narrow 5-15 $\mathrm{Hz}$ band [37]. Baseline correction was performed using the $300 \mathrm{~ms}$ of pre-stimulus activity and data epoched in the range -300 ms to $400 \mathrm{~ms}$. Trials with abnormal activities were excluded based on a detection of extreme values, abnormal trends, and abnormal distributions, using EEGLAB functions $[59,60]$. The threshold for extreme values was $\pm 100 \mu \mathrm{V}$ for all channels. An epoch was rejected for abnormal trend if it had a slope larger than $75 \mu \mathrm{V} /$ epoch and a regression $R^{2}$ larger than 0.3 . An epoch was rejected for abnormal distribution when its kurtosis fell outside five standard deviations of the kurtosis distribution for each single electrode or across all electrodes. All remaining trials were included in the analyses, whether they were associated with correct or incorrect behavioural responses.

Using a multiple linear regression approach, the singletrial EEG amplitude in $\mu \mathrm{V}$ was expressed using one model for all three experiments:

$$
E E G=\beta_{1}+\beta_{2} S+\beta_{3 \phi}+\beta_{4 \gamma 2}+\beta_{5 \gamma 1}+\beta_{6 \phi \gamma 2}+\beta_{7 \phi \gamma 1}+\varepsilon
$$


The fit was performed at each electrode and each time point independently using the glmfit Matlab function, with a normal distribution. Phase $(\phi)$, skewness $\left(\gamma_{1}\right)$ and kurtosis $\left(\gamma_{2}\right)$ were coded as continuous regressors, while the regressor for stimulus identity (e.g., Face A vs. Face B) $(S)$ was a categorical factor. Regression coefficients $(\beta)$ are expressed in an arbitrary unit that reflects the strength of the fit (i.e. the influence of the factor on the EEG signal). The terms $\left(\phi \gamma_{2}\right)$ and $\left(\phi \gamma_{1}\right)$ correspond to, respectively, phase-kurtosis and phase-skewness interactions. The error term is $(\varepsilon)$.

For each subject, we report the electrode at which the model provided the best fit (i.e., where $\mathrm{R}^{2}$ was largest). In general, $\mathrm{R}^{2}$ was largest in a cluster of posterior electrodes that also exhibited large N170 responses to faces. In some cases, the largest $\mathrm{R}^{2}$ was obtained at an electrode that did not produce the strongest N170 to faces. For each of these cases, however, the time-course and relative strength of the best-fitting regression parameters were virtually identical at both sites (i.e., the one producing the largest N170 to faces and the other that produced the largest $\mathrm{R}^{2}$ ). In addition to the multiple intra-subject analyses, we evaluated the influence of each regressor on the EEG across subjects using semi-partial correlation coefficients.

\section{Results}

\section{Experiment I: discrimination between two faces}

In terms of RT, subjects presented one of two patterns. Four out of eight subjects showed an inverted U-shape function, with the shortest RT for extreme values of phase coherence, and the longest RT for ambiguous conditions. Among the other four subjects, three showed longer RT at low phase coherence and a sharp transition towards shorter RT at high phase coherence, while one subject showed the opposite pattern. The RT profiles from these last four subjects were correlated with accuracy (first three subjects: $\mathrm{r}<-.77, \mathrm{p}<.01$; last subject: $\mathrm{r}>.76, \mathrm{p}<.01$ ). This correlation occurred because response accuracy was near chance levels in all subjects when phase coherence was $0 \%$, and increased significantly with greater phase coherence. Figure 3 shows percent correct for each subject as a function of phase coherence. There was a large variability in response patterns across subjects and sessions but all subjects were at or close to ceiling performance at the highest levels of phase coherence.

EEG results are illustrated in Figure 4. The mean ERP was modulated strongly by phase information starting at about $100 \mathrm{~ms}$ after stimulus onset and ending $200 \mathrm{~ms}$ later. The time-course of the explained variance $\left(\mathrm{R}^{2}\right)$ reveals time windows in which the regression model provided a good fit to the data. For all subjects, explained variance started to rise just after $100 \mathrm{~ms}$ and peaked circa 150 $\mathrm{ms}$, at about the same time the N170 peaked in the $100 \%$ phase coherence condition. There was no effect of the stimulus factor: presentation of face 1 or face 2 did not affect EEG amplitude. To confirm this result using a more traditional analysis, we compared systematically the mean single-trial EEG activity to face 1 and face 2, at all electrodes and time points, for each observer. Even with a very liberal univariate strategy (percentile bootstrap, 1000 resamples, $\alpha<.05$, no correction for multiple comparisons), no significant effect was found in any observers. The same non-significant result was obtained when the analysis was performed on a more robust measure of central tendency, the $20 \%$ trimmed mean, rather than the mean. The same approach failed to reveal any significant difference in our two control experiments. Thus, the EEG activity in our experiment was not related to stimulus identity (i.e. face 1 vs. face 2 , or texture 1 vs. texture 2 ).

The EEG signal was strongly modulated by phase coherence and kurtosis, but not skewness. Significant phase effects had a mean onset of $127 \mathrm{~ms}$, kept increasing for about $30 \mathrm{~ms}$ and peaked at $160 \mathrm{~ms}$ after stimulus onset, at about the same time as the N170 (Table 1). More importantly, there was a significant phase $\times$ kurtosis interaction, starting at the transition between the P1 and the N170 components $(150 \mathrm{~ms})$, and reaching a maximum around the peak latency of the N170 (166 ms). There was no significant correlation between N170 latency and any of the model predictors. Phase, kurtosis, and phase $\times$ kurtosis effects where localized around the occipital-temporal electrodes with a maximum $\mathrm{R}^{2}$, specifically around electrodes $\mathrm{PO} 7 / 8$, P9/10, but also O1/2, with a right hemisphere preponderance (7/12 subjects). Table 1 reports maximum $\mathrm{R}^{2}$ statistics in the three experiments. The interaction between phase and kurtosis can be visualized on the modelled data as a stronger phase modulation in the lower image kurtosis range. In other words, an increment in phase coherence had a stronger impact on single-trial amplitude when kurtosis was low compared to when kurtosis was high (Figure 5, top right). For the four subjects that were tested twice, the overall quality of the model and its time course were very consistent (overall correlations at the electrode with max $\mathrm{R}^{2}$ for each subject: $\mathrm{r}=.92, \mathrm{r}=.93$, $\mathrm{r}=.97, \mathrm{r}=.99$, all $\mathrm{p}<.0001$ ). Notably, only one subject, in one of his two sessions, did not show a significant phase $\times$ kurtosis interaction, suggesting that the phase and the phase $\times$ kurtosis interaction are very reliable (testretest).

Across the four subjects, the mean value of the maximum $\mathrm{R}^{2}$ was $36.2 \%$ (median $=35.7 \%$; minimum $=19.8 \%$, maximum $=67.3 \%)$.

The practical relevance of the different predictors was evaluated by the semi-partial variance across subjects. The semi-partial variance is a measure of the unique variance 


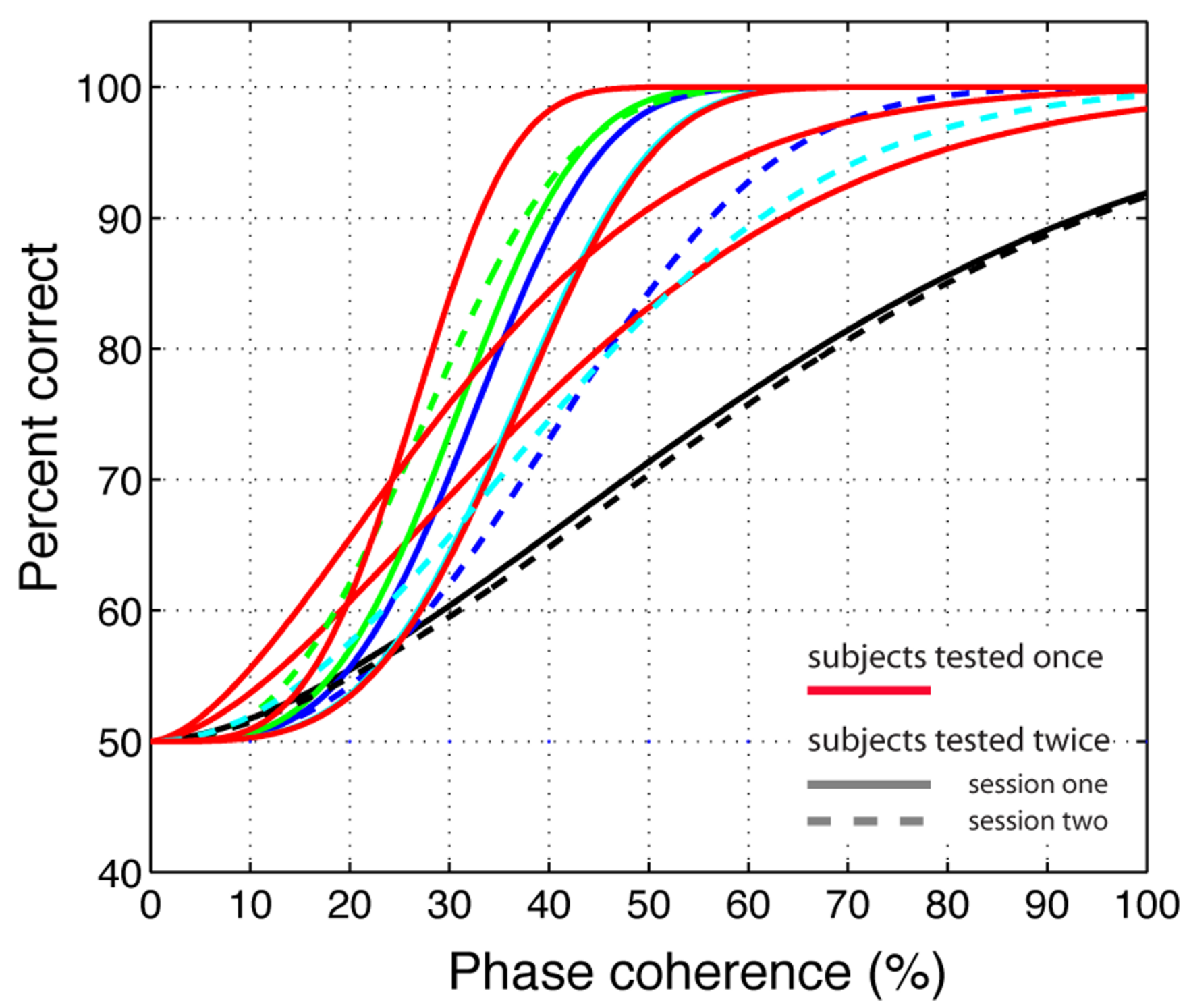

Figure 3

Percent correct for individual subjects in Experiment I. The black lines show data from subject RXL. Subject RXL was singled-out because he is the only subject that was tested in experiment I and the two control experiments. The other colours depict data from all other observers and sessions. Continuous lines indicate the first recording session, while dashed lines indicate the second session of subjects who were tested twice. Data from subjects who were tested only once are indicated in red. Data were fit using a cumulative Weibull function where the accuracy $p$ was expressed as a function of the phase coherence $c$, the phase coherence $\alpha$ supporting $82 \%$ threshold performance, and $\beta$ the slope of the curve: $p=1-0.5 e^{-(c / \alpha)^{\beta}}$

explained by one predictor after controlling for the effects of the other predictors, i.e. all other predictors were partialled out of the predictor of interest (for instance phase), but contrary to the partial variance, they were not partialled out from the dependent variable (our EEG response). The analysis of semi-partial variance revealed the significantly stronger impact of phase coherence on
EEG single-trials, followed by kurtosis and the phase by kurtosis interaction, which did not differ from one another (Figure 6). Stimulus, skewness, and the phase by skewness interaction did not contribute significantly to explaining the EEG variance. This analysis is important, because it shows that both phase and kurtosis have unique effects, i.e. the effect of one cannot be explained 

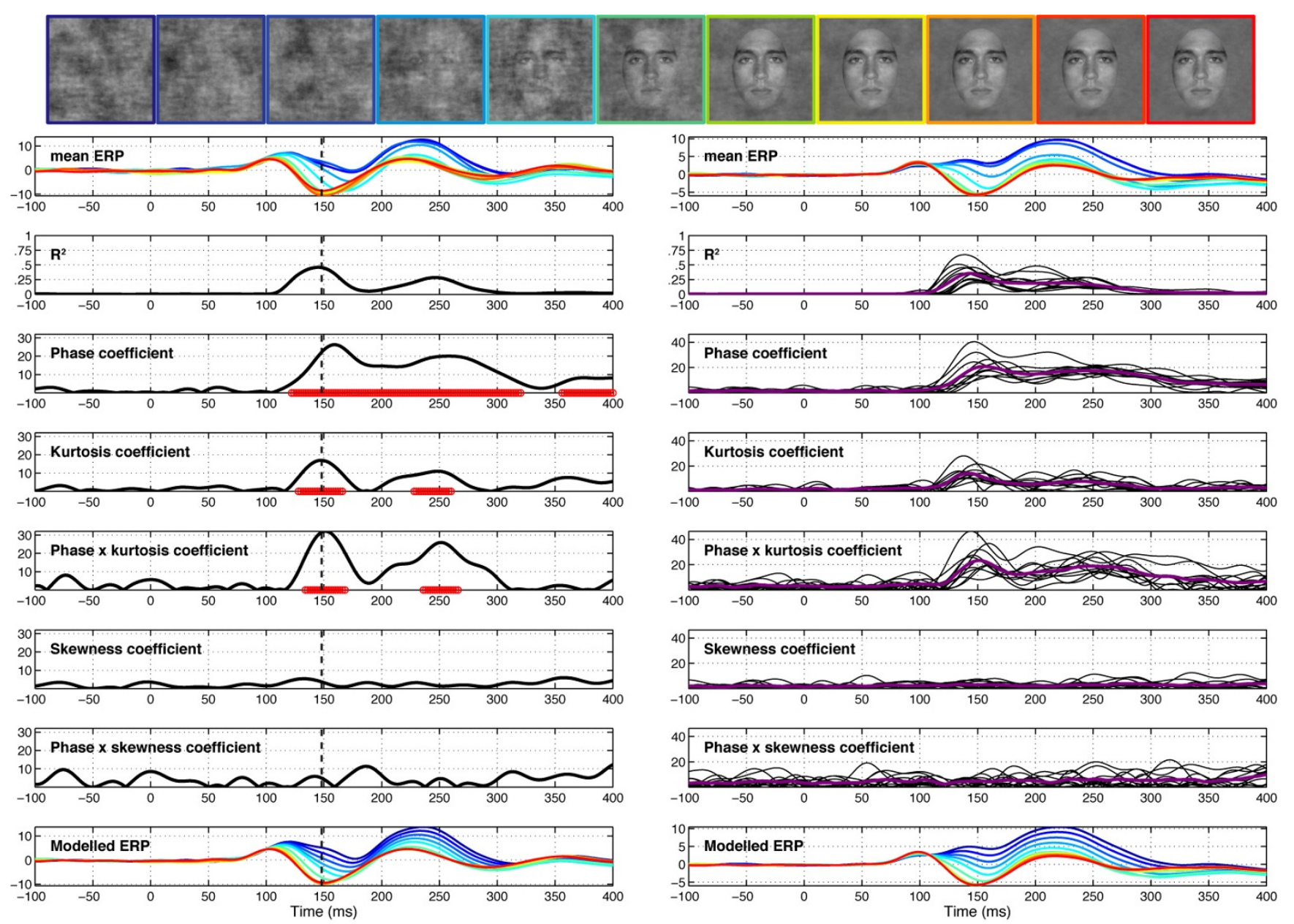

\section{Figure 4}

Session I of subject RXL (left) and data averaged across subjects (right) in Experiment I. The vertical line in the left column indicates the latency of maximum $R^{2}$ for the model, recorded at electrode El07 for RXL, an electrode halfway between T5 and OI. Electrode EIO7 is at the centre of the left red cluster in the topographic map of explained variance in Figure 5. At the top, the row of face stimuli shows the colour code used in the mean ERP and modelled ERP plots, from blue $(0 \%$ phase coherence) to red (100\% phase coherence). The time course of the coefficient for the different model parameters is depicted in black, with the horizontal red line showing periods of statistically significant fitting $(p<.0 \mathrm{I}$, not corrected for multiple comparisons). In the right column, purple lines show the mean coefficient across subjects and the thin black lines data from individual subjects. The beta coefficients are expressed in terms of signal change in $\mu \mathrm{V}$ per unit of the predictor variable.

simply by its linear association with the other (there was no significant quadratic relationship between phase and kurtosis).

Finally, in Figures 4 and 5, a larger P2 component (peaking between 200 and $250 \mathrm{~ms}$ ) is apparent in response to noise patterns ( $0 \%$ phase coherence, in blue) compared to faces $(100 \%$ face coherence, in red), a result that we have observed previously $[28,37]$. To determine if the difference in P2 amplitude was independent from the N170 amplitude, and might therefore reflect a mechanism of interest, a peak-to-peak analysis was carried out on the modelled data (Figure 7). Larger P2 amplitude in the 0\% phase coherence condition, independently of the N170 amplitude, should lead to a decreasing peak-to-peak difference with increasing phase coherence. At $0 \%$ phase coherence, the peak-to-peak difference between the N170 and the P2 was $-8.8 \mu \mathrm{V}$ with a $95 \%$ bootstrap confidence interval of [-10.7-6.9 $\mu \mathrm{V}]$. At $100 \%$ phase coherence, the peak-to-peak distance was very similar, with a mean of $9.2 \mu \mathrm{V}[-10.8-7.5 \mu \mathrm{V}]$. The confidence interval for the difference between the two peak-to-peak differences was narrow and included zero [-0.2 $0.9 \mu \mathrm{V}$ ], thus failing to show a relatively larger $\mathrm{P} 2$ in response to noise textures. If anything, the peak-to-peak difference tended to increase from $0 \%$ up to about $70 \%$ of phase coherence, which goes 
Table I: Model fit results and $95 \%$ confidence intervals in the three experiments

\begin{tabular}{|c|c|c|c|c|}
\hline & & Experiment I: Faces & Experiment 2: Pink Noise Textures & Experiment 3: Wavelet Textures \\
\hline \multirow[t]{4}{*}{$\operatorname{Max} R^{2}$} & Mean Amplitude & $36.4[29.244 .6]$ & $5.8[3.68 .3]$ & 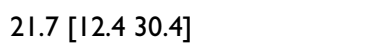 \\
\hline & Min Amplitude & 19.9 & 2.8 & 5.5 \\
\hline & Max Amplitude & 67.4 & 13.1 & 36.6 \\
\hline & Mean Latency & 145 [ 142 | 48$]$ & $250\left[\begin{array}{ll}228 & 272\end{array}\right]$ & 229 [ 180.8296 .6$]$ \\
\hline \multirow[t]{3}{*}{ Phase } & Onset Latency & $127\left[\begin{array}{lll}12 \mid & 134\end{array}\right]$ & NA & $218[161263]$ \\
\hline & Peak Latency & $160[154 \quad 168]$ & NA & 228 [ 175 269] \\
\hline & Onset-Peak Latency Difference & $33[2642]$ & NA & $10\left[\begin{array}{lll}6 & 14\end{array}\right]$ \\
\hline \multirow[t]{3}{*}{ Kurtosis } & Onset Latency & $127\left[\begin{array}{lll}122 & 132\end{array}\right]$ & NA & 175 [163 199] \\
\hline & Peak Latency & $143\left[\begin{array}{lll}137 & \mid 48\end{array}\right]$ & NA & $204\left[\begin{array}{lll}187 & 219\end{array}\right]$ \\
\hline & Onset-Peak Latency Difference & $16\left[\begin{array}{lll}13 & 19\end{array}\right]$ & NA & $29\left[\begin{array}{ll}20 & 42]\end{array}\right.$ \\
\hline \multirow[t]{3}{*}{ Phase $\times$ Kurtosis } & Onset Latency & $150\left[\begin{array}{lll}133 & 17 \mid\end{array}\right]$ & NA & NA \\
\hline & Peak Latency & $166\left[\begin{array}{lll}151 & 185\end{array}\right]$ & NA & NA \\
\hline & Onset-Peak Latency Difference & $16\left[\begin{array}{ll}12 & 20\end{array}\right]$ & NA & NA \\
\hline
\end{tabular}

$R^{2}$ values are expressed as percentages. Latencies are expressed in ms. Regarding max $R^{2}$, all the pairwise comparisons were significant (percentile bootstrap, 1000 resamples): Experiment I vs. Experiment 2, mean difference 30\% [22 39], p = 0; Experiment I vs. Experiment 3, mean difference $15 \%$ [3 27], $\mathrm{p}=.009$; Experiment 2 vs. Experiment 3, mean difference $16 \%$ [5 25], $\mathrm{p}=.002$. Phase, Kurtosis, and Phase $\times$ Kurtosis interaction regressor time courses were analyzed at the electrode showing the max $\mathrm{R}^{2}$ for each subject individually. Onset Latency refers to the earliest time at which a given model parameter contributed significantly to explaining the EEG data. NA stands for non-applicable, which corresponds to situations where only one subject showed a significant effect (Experiment 3) or the values are meaningless because of the poor model fit and the lack of significant effects (Experiment 2)

against the idea that the difference between N170 and P2 amplitude might be reduced with increasing phase coherence. Therefore, it seems that the P2 difference is a simple carry-over of the N170 effect.

The results of Experiment 1 indicate that the event-related response to faces was not sensitive to face identity in our task. The main determinant of ERP variations was the structure of the stimuli, as captured by global phase coherence and kurtosis. To ensure that this was the case, a control experiment was conducted, in which the task was identical to the one employed in Experiment 1, but the target stimuli were pink noise textures created by randomizing completely the phase of the original face stimuli (Figure 8). This manipulation insured that the textures had the same amplitude spectrum as the faces used in Experiment 1, and that all textures had almost the same global statistics (Gaussian pixel histogram, constant kurtosis 3) across all levels of phase coherence, while allowing subjects to perform the original task.

\section{Experiment 2: discrimination of two pink noise textures} Although the textures used in Experiment 2 appear, at first glance, to be very similar, response accuracy in Experiment 2 was similar to the performance obtained with faces in Experiment 1 (Figure 9).
Although behavioural performance was very good in this task, pink noise textures failed to elicit the EEG pattern of sensitivity to phase noise evoked by faces in Experiment 1 (Figure 10). The global explained variance was extremely low $(5.8 \%)$ compared to the high values previously obtained $(36.4 \%)$, showing that our model failed to describe the data properly (Table 1 ). This result was true for all subjects, despite the fact that the pink noise textures elicited a very strong evoked response in the time range 150-250 ms post stimulus onset (Figures 10, 11 and 12). Even after extensive training with the same set of two stimuli there was no evidence of sensitivity to phase noise: Figure 12 shows data from a regular EEG session after 4408 trials, including previous EEG sessions and further behavioural practice between EEG recordings. Even in this case, the model provided a relatively poor fit of the data ( $\max$ $\mathrm{R}^{2}=13.1 \%$ ). In addition, that fit was delayed by at least $100 \mathrm{~ms}$ compared to the best fit obtained for faces in Experiment 1 . Thus, it seems that the pattern of sensitivity to phase noise observed in response to faces is not related to task performance, i.e. subjects' capacity to discriminate between Stimulus A and Stimulus B, but might rather depend on the structure of the image.

\section{Experiment 3: discrimination between two wavelet noise textures}

Finally, we performed a last control experiment to determine whether textures with the same higher-order image 

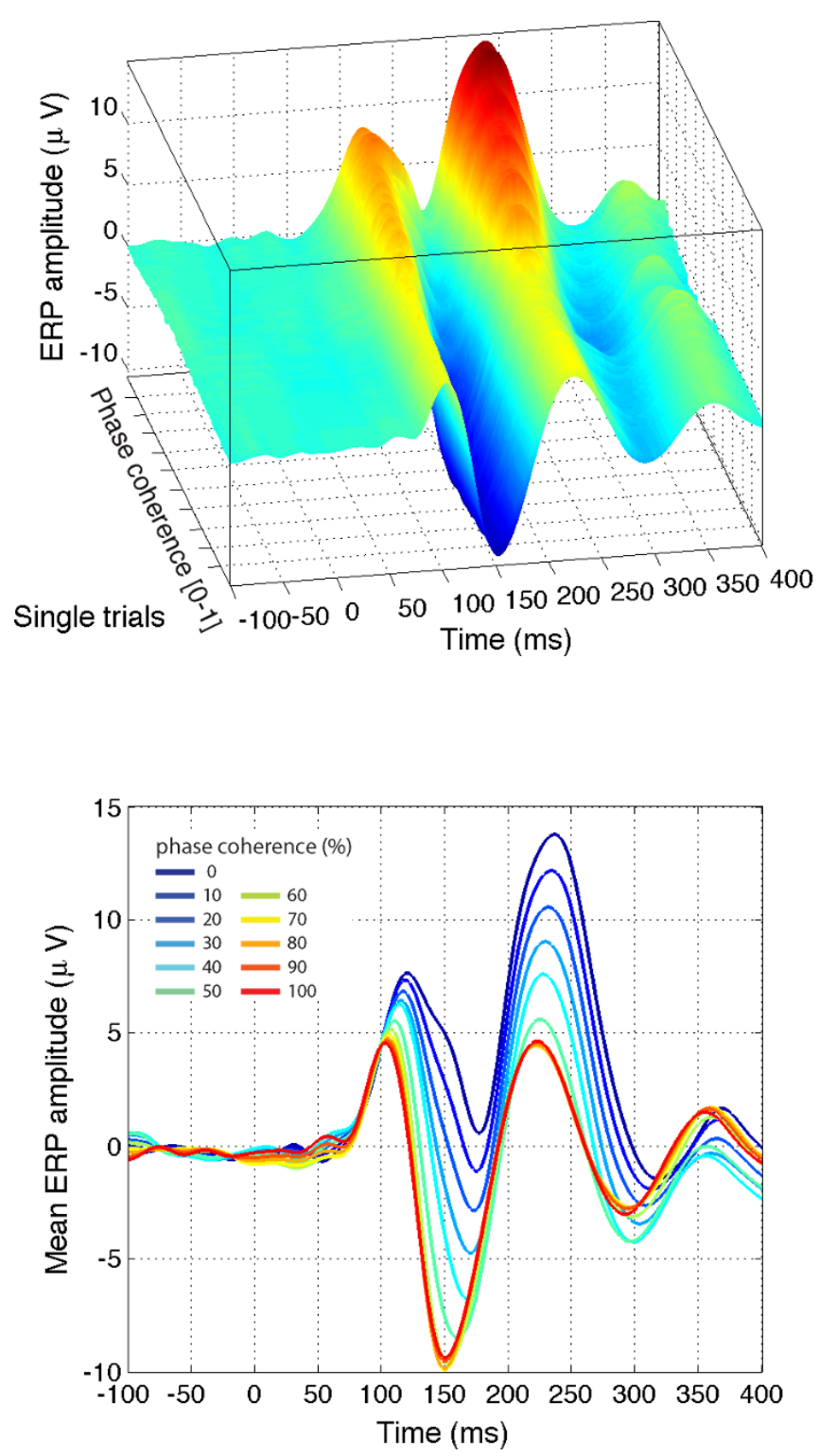

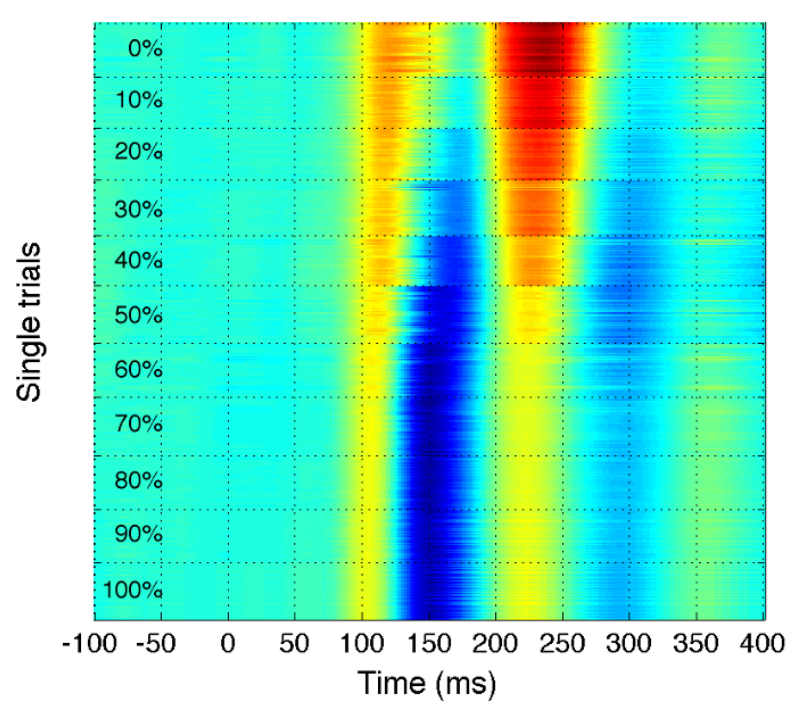

Original 100\% phase coherence ERP Explained variance
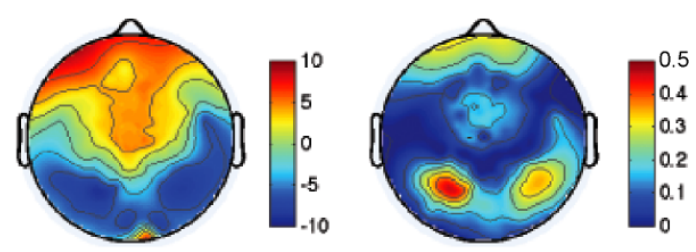

\section{Figure 5}

Modelled data and topographical maps from session I of subject RXL in Experiment I. The upper part of the figure shows 3D (left) and 2D (right) representations of single trials, sorted by chronological order in which they were recorded during the experiment, independently for each bin of phase coherence. In the lower left corner, single-trial modelled data were averaged according to phase coherence level, and colour coded from blue (0\%) to red (100\%) following Figure 4 nomenclature. The data are from the electrode at which the maximum $\mathrm{R}^{2}$ was obtained. The topographic maps show the interpolated ERP signal (left, in $\mu \mathrm{V}$ ) and explained variance (right, in \%) at the latency of maximum $\mathrm{R}^{2}$ (I48 ms). The electrode showing the best fit is at the centre of the lower left red cluster in the explained variance map. For this subject, the electrode showing the maximum NI70 was over the right hemisphere. However, at this electrode, the pattern of model fit was virtually indistinguishable from the one showed here.

statistics as faces were sufficient to elicit the pattern of sensitivity to phase noise observed in Experiment 1. Unlike the pink noise textures used in Experiment 2, the kurtotic textures created for Experiment 3 did contain local elements (edges and 'blobs') that were similar to those seen in faces (Figure 13). With these textures, behavioural performance was very good for all subjects (Figure 14).

Importantly, EEG activity showed a clear N170 effect, with a much stronger signal for $100 \%$ phase coherence stimuli compared to $0 \%$ phase coherence stimuli between 150 

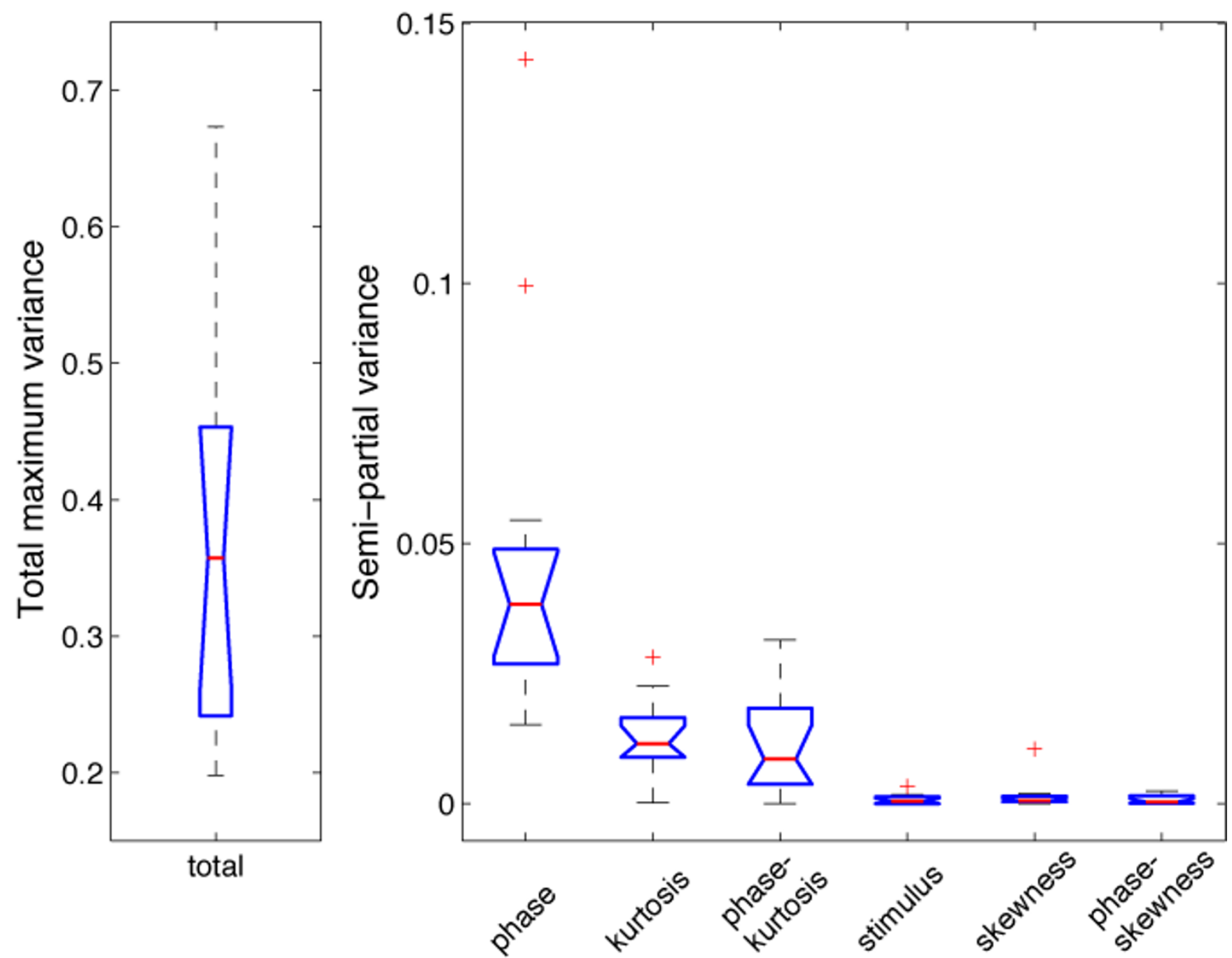

\section{Figure 6}

Boxplots of total maximum explained variance and semi-partial variance in Experiment I. For each subplot, the red line indicates the median. The blue box extends from the upper to the lower quartile values. The whiskers show the most extreme points that are within 1.5 times the inter-quartile range. A red plus is an outlier. The notch in the blue box corresponds to a robust estimate of the median confidence interval. Non-overlapping notches indicate that medians differ with $95 \%$ confidence. Because of the scaling involved in the computation of the semi-partial variance, the sum of semi-partial variances across regressors is less than the total explained variance. The semi-partial variance calculation was performed at the electrode and time point of maximum $\mathrm{R}^{2}$ peak for each subject.

and $200 \mathrm{~ms}$ after stimulus onset. This response pattern contrasted sharply with the response generated by pink noise textures, showing no N170 effect, and a very poor model fit overall. This activity was modulated by phase coherence in three subjects out of five (Figures 15 and 16). However, despite some similarities, the EEG response to wavelet textures did not match completely the response triggered by faces. Particularly, the model provided a lower and delayed fit of the data compared to Experiment 1 (Table 1). Furthermore, the phase $\times$ kurtosis interaction was significant in only one of the subjects (onset $=163$ $\mathrm{ms}$, peak latency $=205 \mathrm{~ms}$ ). Finally, an analysis of the semi-partial variance (similar to the one presented in Figure 5) revealed a very weak unique contribution of phase ( $\min =0, \max =0.009)$ compared to the one observed for faces $(\min =0.015$, $\max =0.143)$, and no significant difference among the unique contributions of the different regressors $\left(F_{(5,20)}=2.1, P=0.4\right)$. Although limited by the small number of subjects tested in our second control experiment, these results suggest that higher-order image 


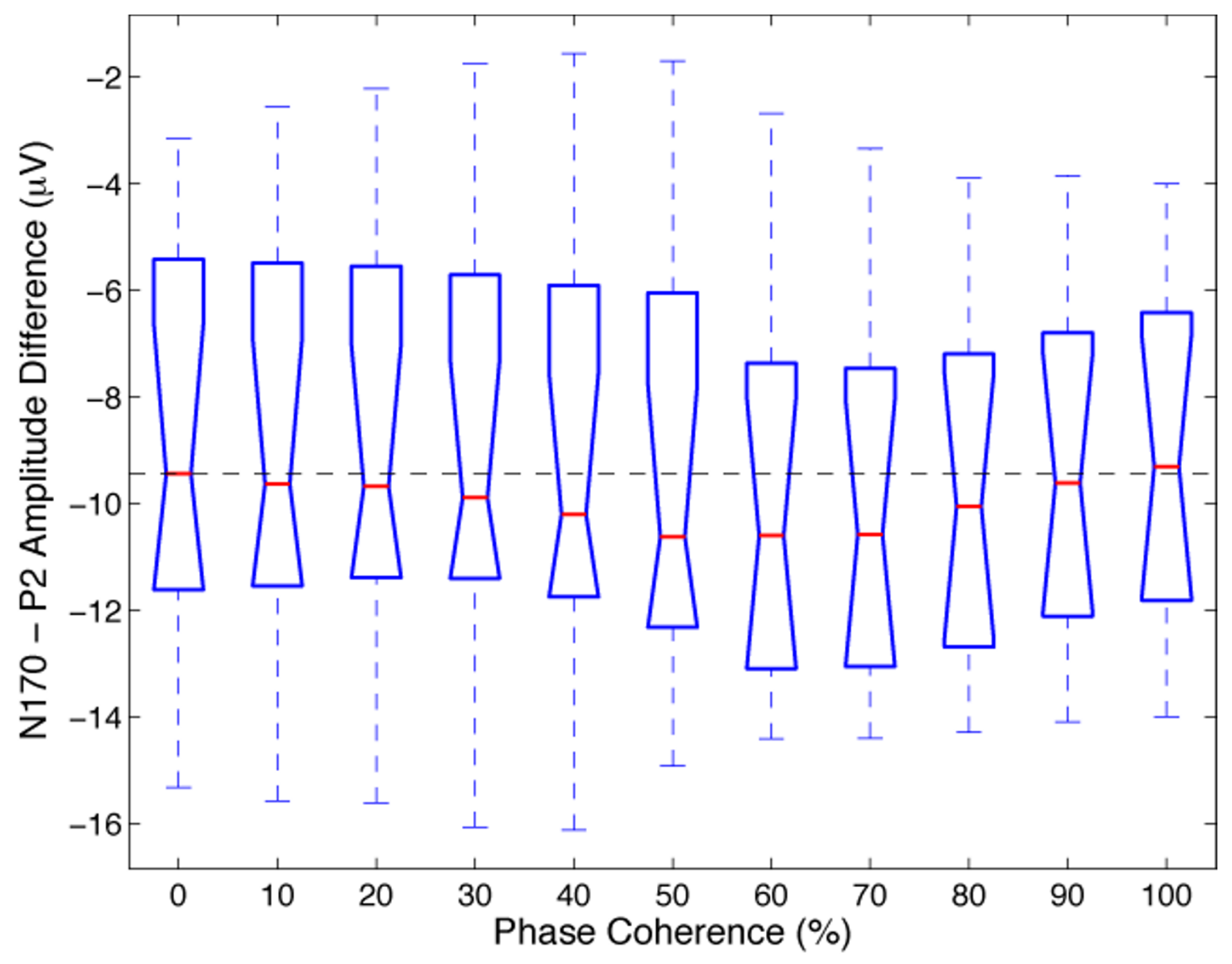

Figure 7

Boxplot of the peak-to-peak differences between the NI70 and the P2 measured on modelled data at different levels of stimulus phase coherence for all subjects and sessions in Experiment I.

statistics of natural objects, in this case faces, are necessary but not sufficient to explain the pattern of EEG sensitivity to phase information observed for faces. A direct comparison between the sensitivity to phase noise of faces and other stimulus categories, like wavelet textures, will require further testing using a parametric and categorical mixed design.

\section{Discussion}

Our previous work showed that early evoked brain activity to faces and objects, starting at about 130-150 ms after stimulus onset, does not reflect differences in stimulus amplitude spectra, but rather is mainly driven by spatial phase information $[28,36,37]$. The current results reveal for the first time the time course of the brain sensitivity to global visual phase information. In other words, we determined how phase information shapes early brain responses to complex objects like faces. Our results show that the visual system sensitivity to global phase information emerges progressively in a short time window between the P1 and the N170 components. This sensitivity to phase noise starts roughly at about $120-130 \mathrm{~ms}$ after stimulus onset and continues for another $25-40 \mathrm{~ms}$, as indicated by the time course of model $\mathrm{R}^{2}$ and the regression fit (Figure 4). During this delay, single-trial activity is not only sensitive to phase information, but also to kurtosis, and the two global image descriptors interact significantly with each other (Figures 4 and 5). 

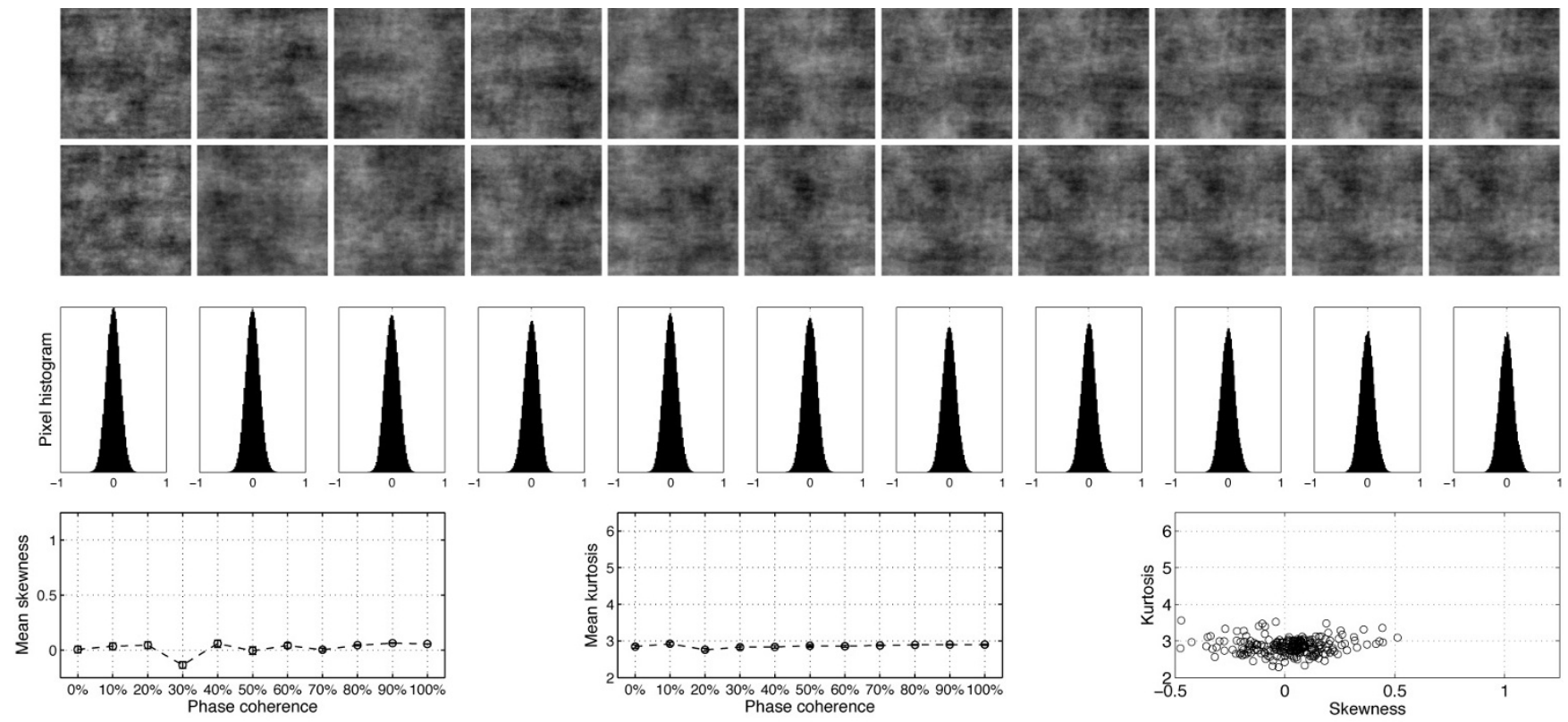

Figure 8

Examples of stimuli used in Experiment 2. The first two rows show the 22 stimuli presented to one observer during the first block of the experiment. The observer discriminated between the same two textures during the whole experiment. The noise level varied from $100 \%$ (left side; $0 \%$ phase coherence) to $0 \%$ (right side; $100 \%$ phase coherence). Histograms in the third row show the distribution of pixel contrasts averaged across all stimuli seen by this observer at each level of phase coherence. Note that, unlike the stimuli used in Experiment I, the pixel histograms had a relatively constant Gaussian distribution across all levels of phase coherence. This constant histogram distribution is depicted in the last row, showing the mean skewness (left), and mean kurtosis (middle), as a function of phase coherence. The error bars correspond to $95 \%$ confidence interval computed using a bootstrap percentile technique (1000 resamples). In the bottom right end graph, kurtosis for each image (each circle) is expressed as a function of skewness. There was no relationship between the two statistical descriptors.

Our linear phase manipulation introduced non-linear monotonic changes in higher-order image statistics, as revealed by kurtosis measurements [46]. Kurtosis is a good measure of image sparseness [50,52,61]. The monotonic and non-linear increase in kurtosis, from $0 \%$ to $100 \%$ phase coherence, thus most probably corresponds to the build up of local elements like edges and contours, that in turn are formed by local phase alignment across different spatial frequency bands $[38,40,50]$. The time course of our model fit might thus reveal the extraction of global image structure, and not only sensitivity to phase information.

We note that our kurtosis measurements were made directly on the pixel contrasts. Thomson [50] warned about measuring kurtosis from non-whitened images because coloured noise (in our case pink noise corresponding to the $0 \%$ phase coherence condition) contains pixel-wise correlations that might inflate kurtosis artificially, rendering it a non-interesting measure to detect sparseness in images. However, we were interested in the relative differences in kurtosis across image types, and, more importantly, our pink noise textures were appropri- ately centred around 3, the value expected from white noise distribution, not contaminated by $1 / \mathrm{f}$ amplitude spectrum information.

Two control experiments (Experiments 2 and 3 ) showed that higher-order statistics of faces are necessary but not sufficient to obtain the sensitivity to phase noise observed in response to faces (Experiment 1). First, although in Experiment 2 subjects' could discriminate two pink noise textures presented in the same conditions as faces in Experiment 1, the regression model failed to fit the data. Second, wavelet textures matched for both global and some of the local image face statistics did trigger a face-like EEG pattern, but in this case the model provided a poorer overall fit to the data, a delayed timing in the fit, and an absence of phase $\times$ kurtosis interaction. This result points to particular local phase arrangements as being responsible for the model fit observed for faces. This is not a trivial point, because it would be conceivable to observe a timecourse of phase noise sensitivity for control textures that would be similar to the one observed for faces. This possibility stems from the fact that the linear regression fit used to measure sensitivity to phase noise is independent of the 


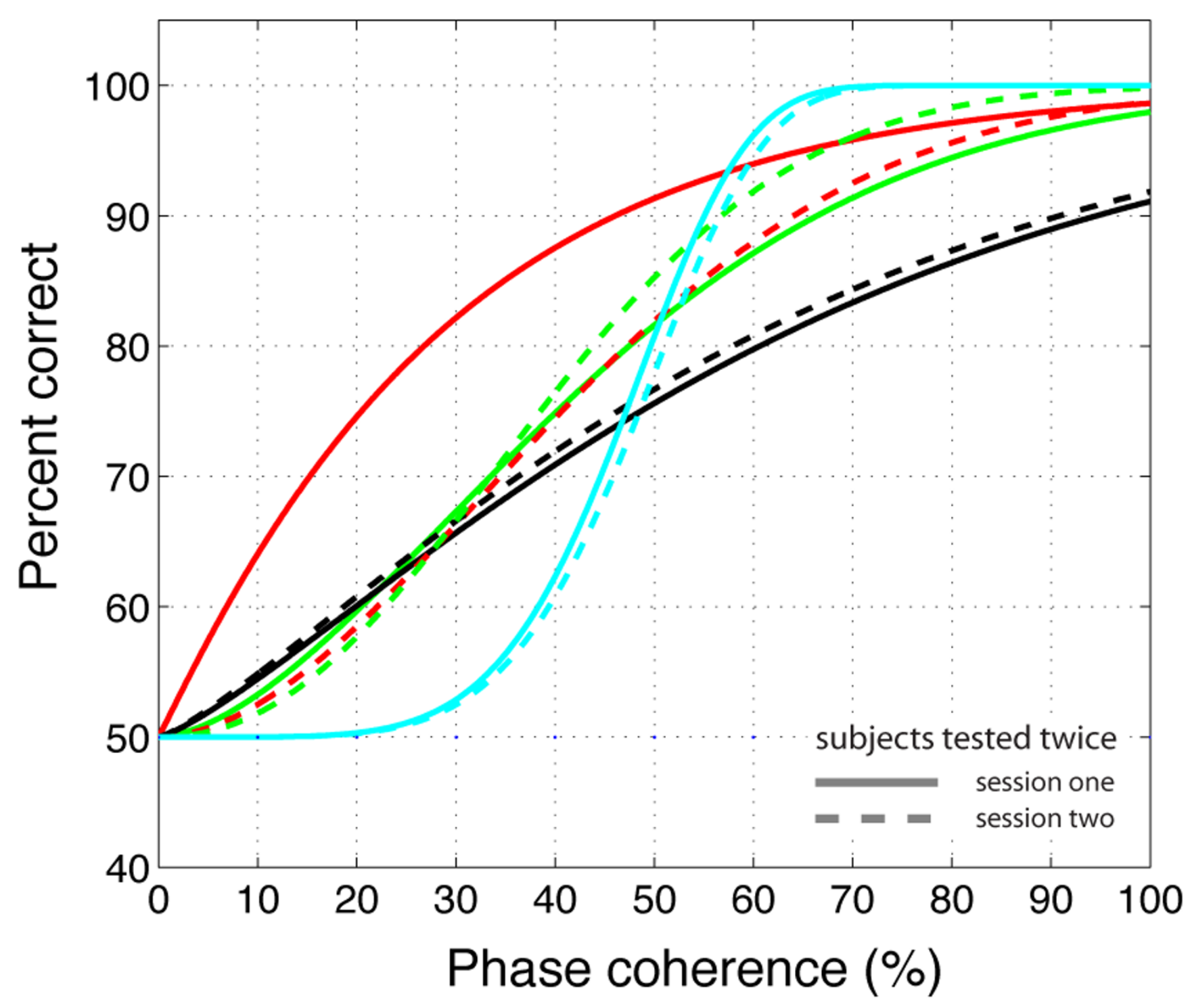

Figure 9

Percent correct for individual subjects in Experiment 2. The black lines show data from subject RXL. The other colours depict data from all other observers and sessions. Continuous lines indicate the first recording session, while dashed lines indicate the second recording session.

global shape of the ERP, i.e. its mean. Our second control experiment also raises the question of how far we can go into matching image statistics without simply reproducing the stimulus. From our results, it remains unclear how the EEG to faces and matched wavelet textures precisely compare within subjects, and this will require further investigation using a paradigm in which the two types of stimuli, as well as control object categories, are tested in the same recording session.

Parametric stimulus phase manipulations and, more generally, parametric noise manipulations have been used in the literature to investigate the spatial and temporal hierarchical encoding of visual information. In the spatial domain, fMRI has lead to the discovery of various noise tuning functions in different brain areas of human and non-human primates. The general conclusion from those studies is that there is an increasing sensitivity to noise along the ventral pathway, from V1, where for instance the signal evoked by natural images does not differ from the one evoked by pink noise $[9,62]$, but see [63], to higher-level object processing areas, where noise sensitivity tends to be the strongest $[7,9,63,64]$. The strongest noise sensitivity in higher levels of the visual hierarchy as 

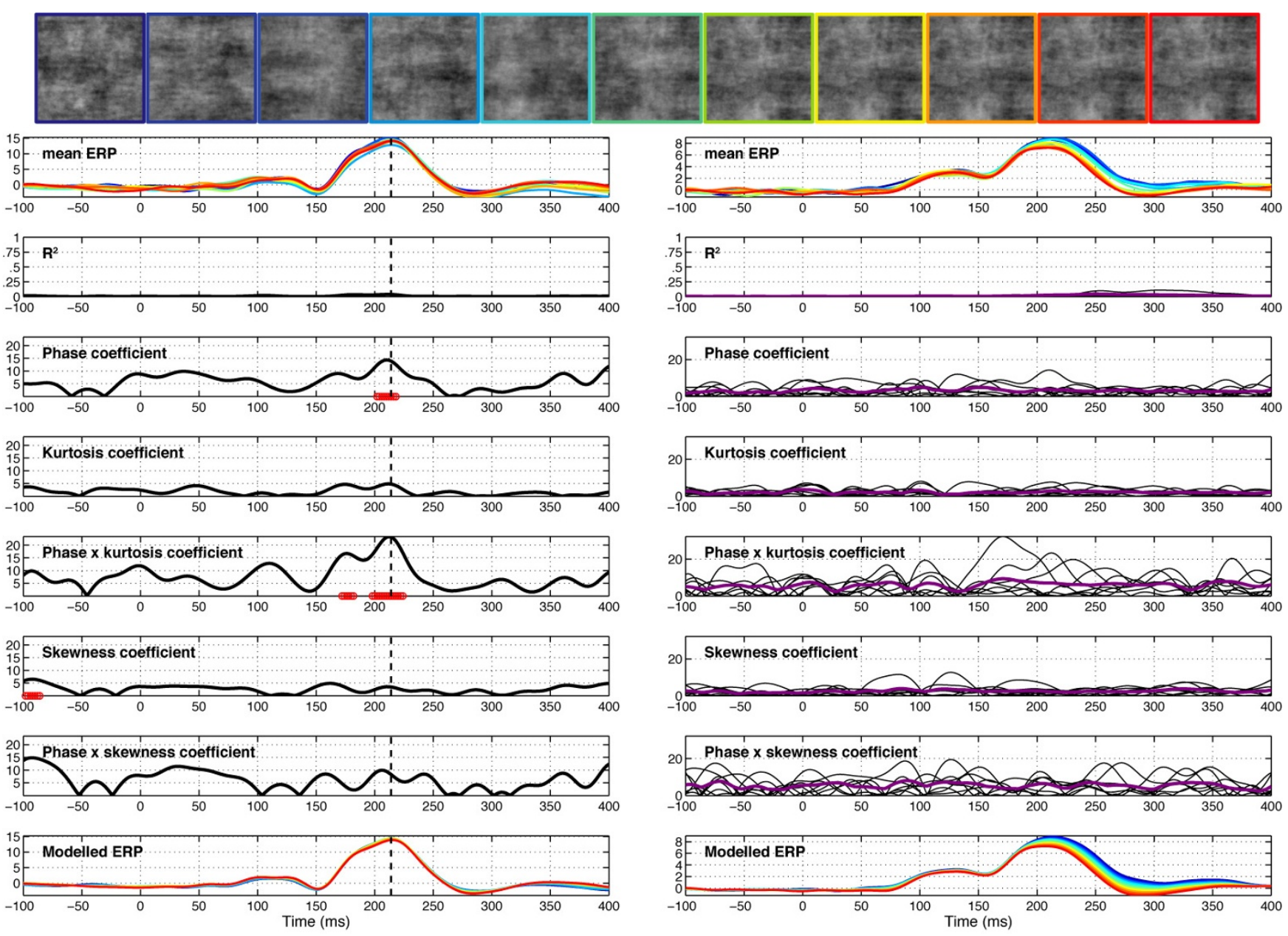

Figure 10

Session I of subject RXL (left) and data averaged across subjects (right) in Experiment 2. Figure caption details are otherwise identical to those of Figure 4.

observed in fMRI, together with EEG and MEG source analyses of evoked activity in the time range of the $\mathrm{N} /$ M170 $[16,65,66]$, suggests that the sensitivity to phase noise we recorded in response to faces corresponds to the activity of face or object processing areas integrating information about the global structure of the stimulus. Future studies should investigate the cortical network involved in the effects reported here, as well as the nature of these effects, essentially feedforward or reflecting the integration of information from other structures $[67,68]$.

In the temporal domain, EEG and MEG studies have reported results compatible with our findings. For instance, additive noise has been used to dissociate the stimulus sensitivity of early evoked responses. In EEG, it has been demonstrated that there is a linear inverse relationship between the amount of white noise added to face stimuli and the N170 amplitude. In contrast, the earlier P1 component is not affected by this noise manipulation
[29]. In MEG, another type of dissociation has been reported between the $\mathrm{M} 1$ and the M170, which are to some extent the magnetic counterparts of the P1 and N170 ERP components [66]. In a parametric design in which faces were masked by narrow band-pass filtered noise, Tanskanen et al. found that the M170 amplitude was modulated by noise spatial frequencies in a very similar manner to recognition performance [32]. When noise patterns were presented in the absence of face stimuli, the spatial frequency noise sensitivity of the M170 disappeared, whereas the earlier M1 component showed frequency tuning similar to the one triggered by face + noise stimuli. It thus seems clear from these two studies that the $\mathrm{N} 170$ reflects, at least in part, the activity of a mechanism that begins to respond during the N170 time window and which was not active during earlier time frames.

In our study, we made the explicit assumption that this mechanism might be related to global phase processing. 

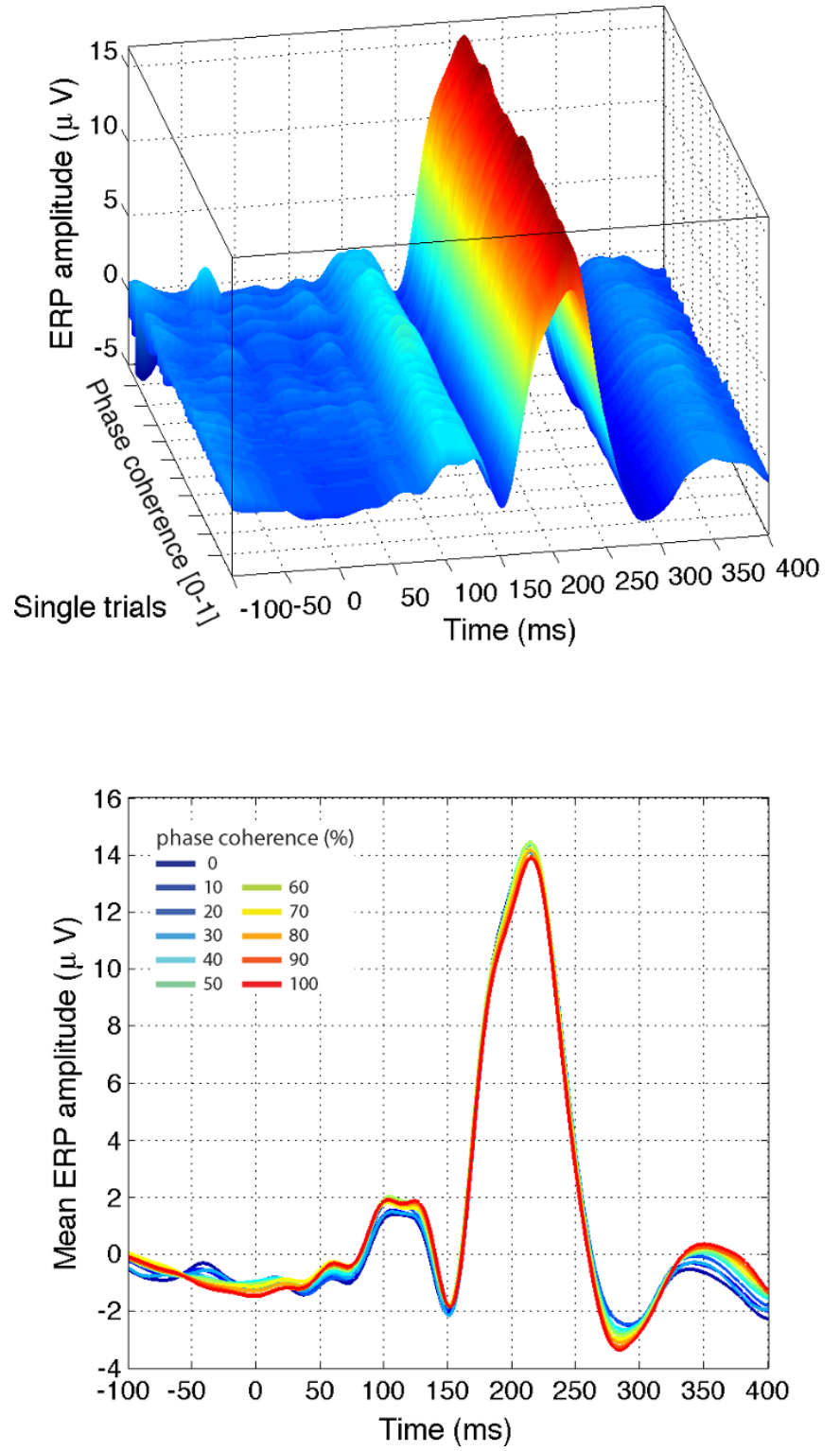

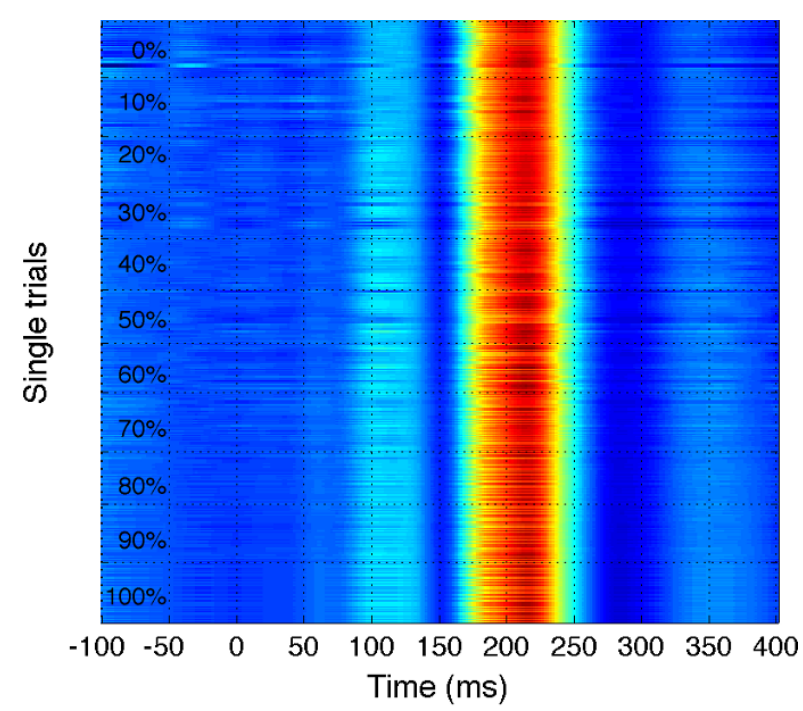

Original 100\% phase coherence ERP Explained variance
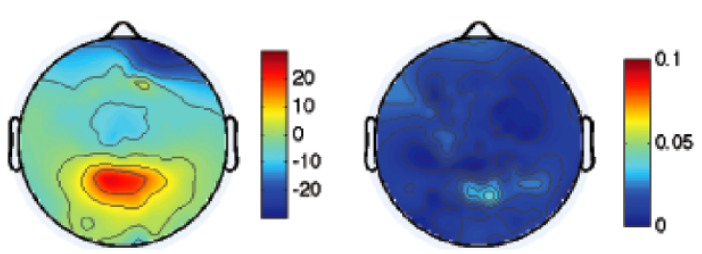

\section{Figure I I}

Modelled data and topographical maps from session I of subject RXL in Experiment 2. The topographic maps show data at the latency of maximum $R^{2}(2 / 4 \mathrm{~ms})$. Figure caption details are otherwise identical to those of Figure 5.

We also provide for the first time a detailed timing of sensitivity to phase noise, using a single-trial model incorporating only parameters related to the global image statistics. Our approach did not depend on the identification of traditional ERP components, and therefore allowed us to track sensitivity to phase noise without limiting our analyses to ERP peaks. This aspect of the analyses is important, because we found that information extraction starts at the transition between the P1 and the N170. This result is in keeping with a series of recent studies, relying on component-free single-trial models, showing onsets of task related information accrual just before the N170, but after the P1 $[14,30,31,69]$.

\section{Conclusion}

Our approach was not to map the relationship between behaviour and EEG activity, but rather to focus on global image properties and how they shape early EEG activity. This is why we kept the task simple and constant. This approach is legitimate because early brain activity evoked by some categories of complex objects, like faces and words, is hardly modulated by task factors $([27,28]$, but see some recent advances in $[14,69])$, and because there is 

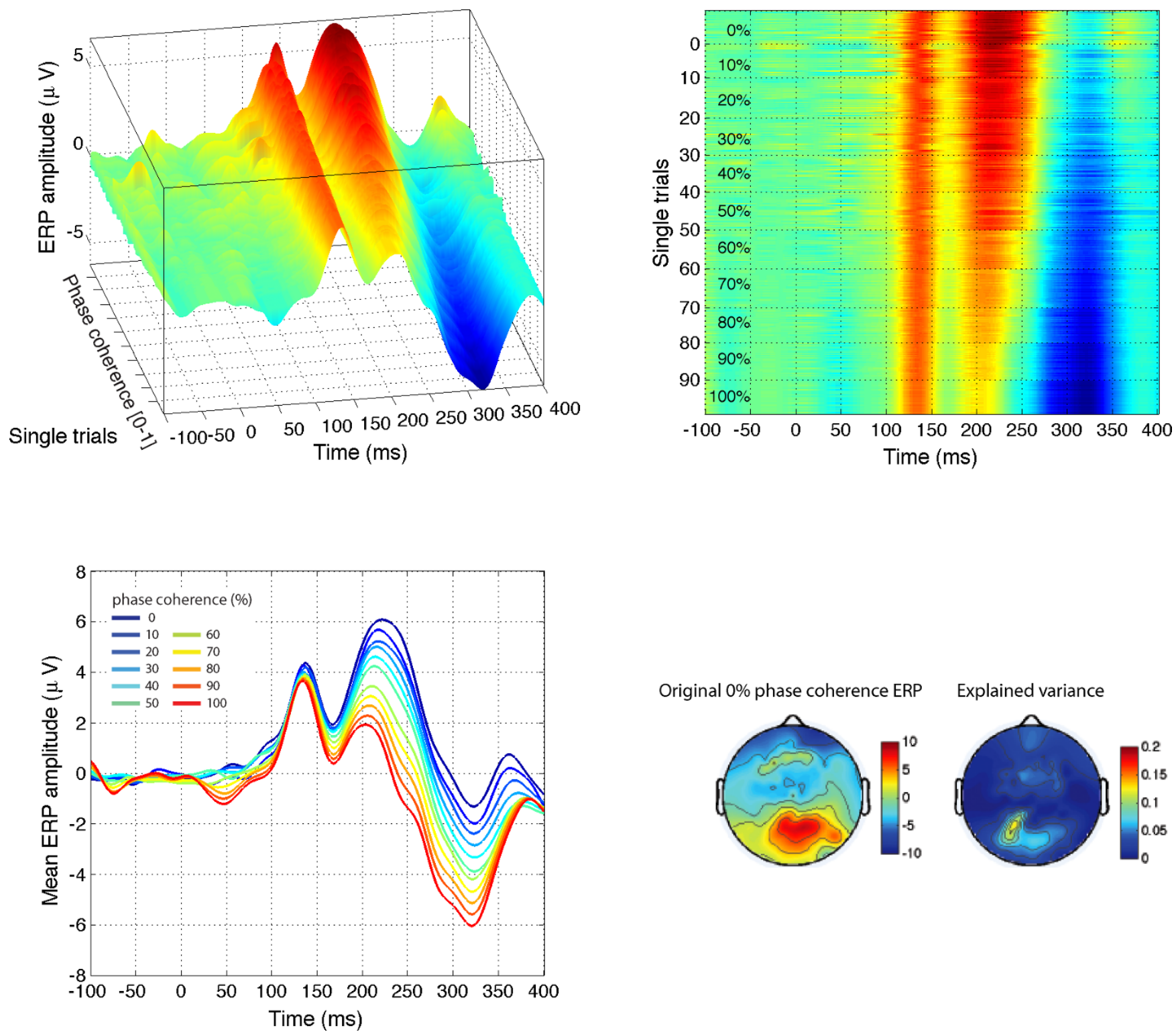

\section{Figure 12}

Third EEG session from subject GAR in Experiment 2. The topographic maps show data at the latency of maximum $\mathrm{R}^{2}$ (242 $\mathrm{ms})$. It appears that data from this subject were particularly sensitive to phase information, as shown by the gradient of amplitude responses in the modelled data. However, the largest amount of variance explained was relatively low $\left(R^{2}=0.13\right)$. Moreover, during the time range 200-300 ms, when the model provided the best fit to the data, none of the regressors were associated significantly with the EEG signal. Only at about $350 \mathrm{~ms}$, a few time points show a significant phase by kurtosis interaction, but in this latency range, the maximum $R^{2}=0.05$.

still much to learn about the relationship between image statistics and brain activity [61]. Furthermore, our approach provides a potentially fruitful departure from frameworks that make assumptions about stimulus space and the nature of relevant 'features'. Like natural scenes, faces can receive a statistical description rather than a category label. For instance, contrasting faces and houses can tell us a great deal about when and where in the brain these two kinds of stimuli are differentially represented. However, other properties co-vary with these semantic categories, and it remains unclear precisely what type of information is extracted when a categorical difference is observed. Using parametric designs can circumvent this limitation. In that framework, phase manipulations con- 

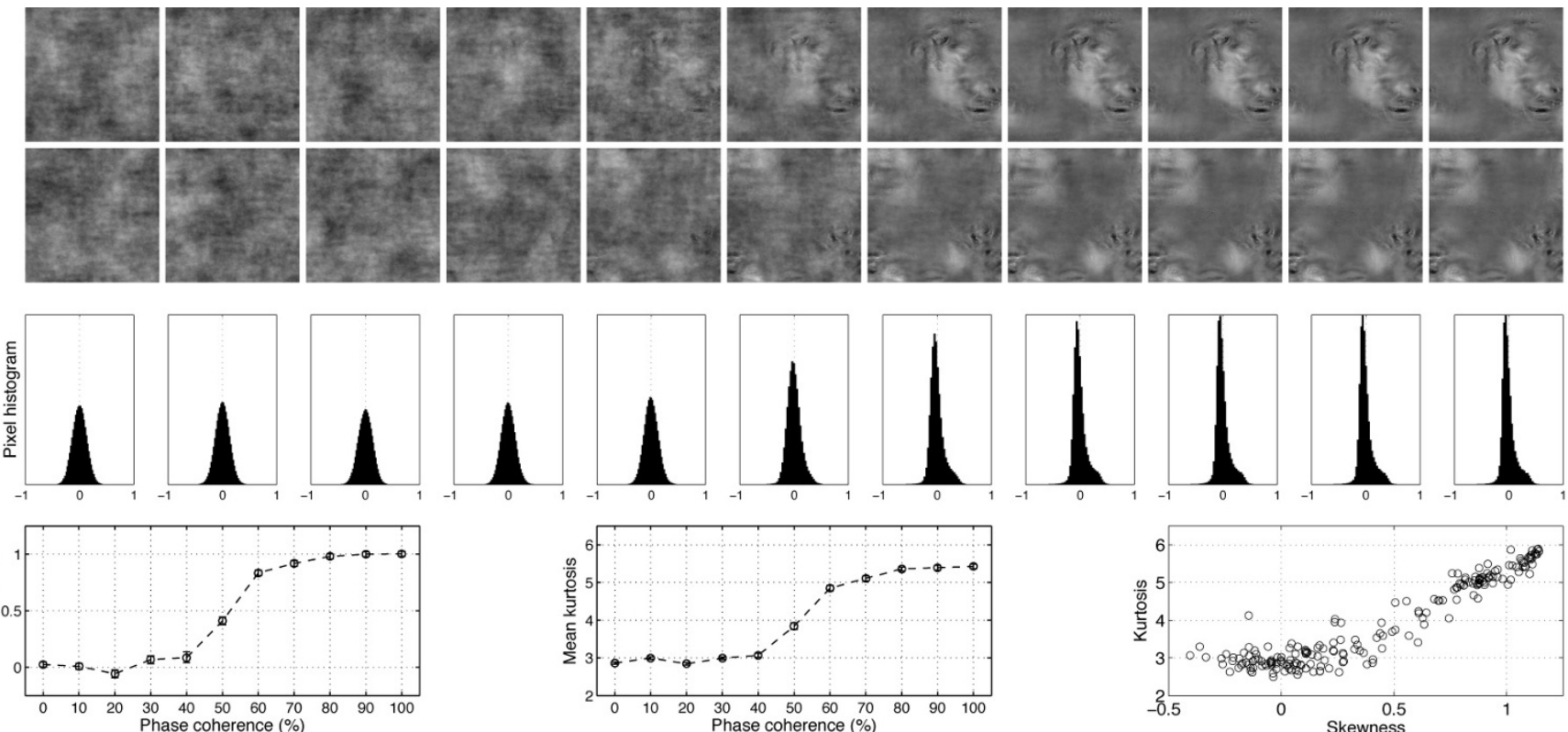

Figure 13

Examples of stimuli used in Experiment 3. The first two rows show the 22 stimuli presented to one observer during the first block of the experiment. The observer discriminated the same two wavelet textures during the whole experiment. The noise level varied from 100\% (left side; $0 \%$ phase coherence) to $0 \%$ (right side; 100\% phase coherence). Histograms in the third row show the distribution of pixel contrasts averaged across the entire set of stimuli seen by this observer at each level of phase coherence, from $0 \%$ (left) to $100 \%$ (right). Similarly to what was observed for faces in Experiment I, and contrary to the pink noise textures used in Experiment 2, the pixel histograms showed a non-linear transition from a Gaussian distribution to a skewed and kurtotic distribution with increasing levels of phase coherence. This progression is depicted in the last row, showing the mean skewness (left), and mean kurtosis (middle), as a function of phase coherence. The error bars correspond to $95 \%$ confidence interval computed using a bootstrap percentile technique (I 000 resamples). In the bottom right end graph, kurtosis for each image (each circle) is expressed as a function of skewness. Similarly to face stimuli, the two statistical descriptors are non-linearly related to one another.

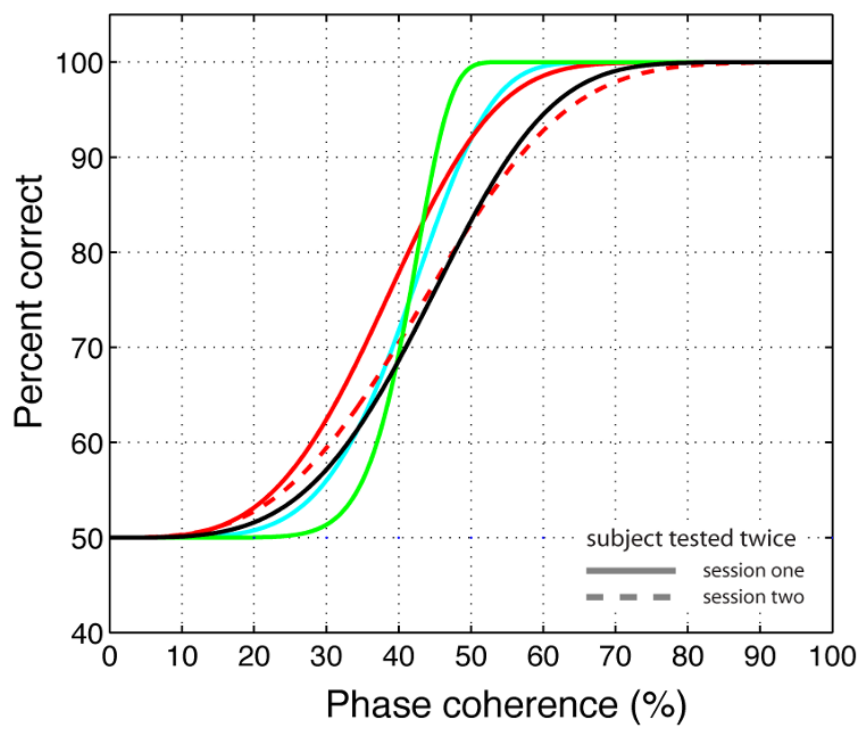

Figure 14

Percent correct for individual subjects in Experiment 3. The black lines show data from subject RXL. The other colours depict data from all other observers and sessions. Continuous lines indicate the first recording session, while dashed lines indicate the second recording session. 

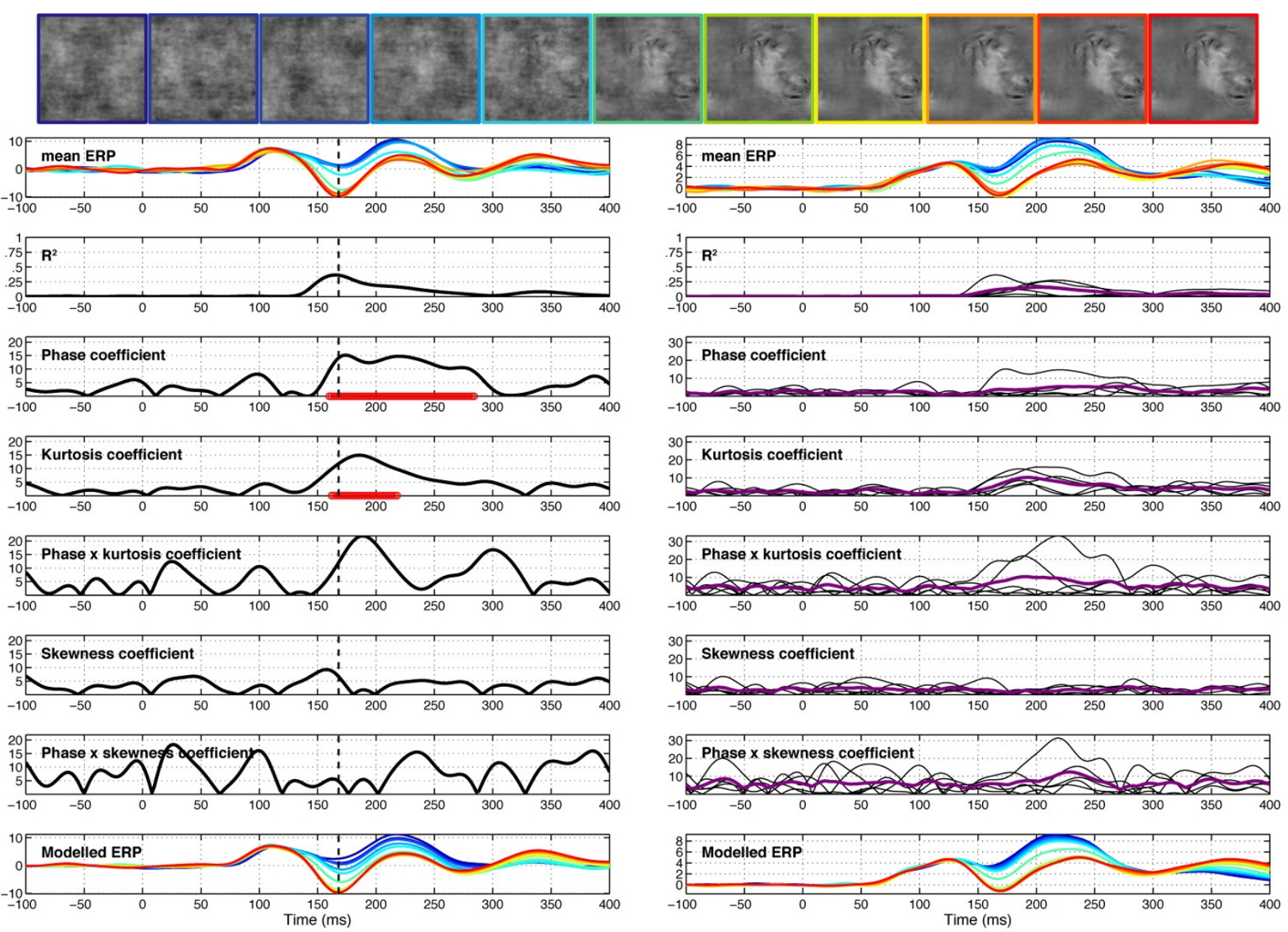

Figure 15

Session I of subject RXL (left) and data averaged across subjects (right) in Experiment 3. Figure caption details are otherwise identical to those of Figure 4. 

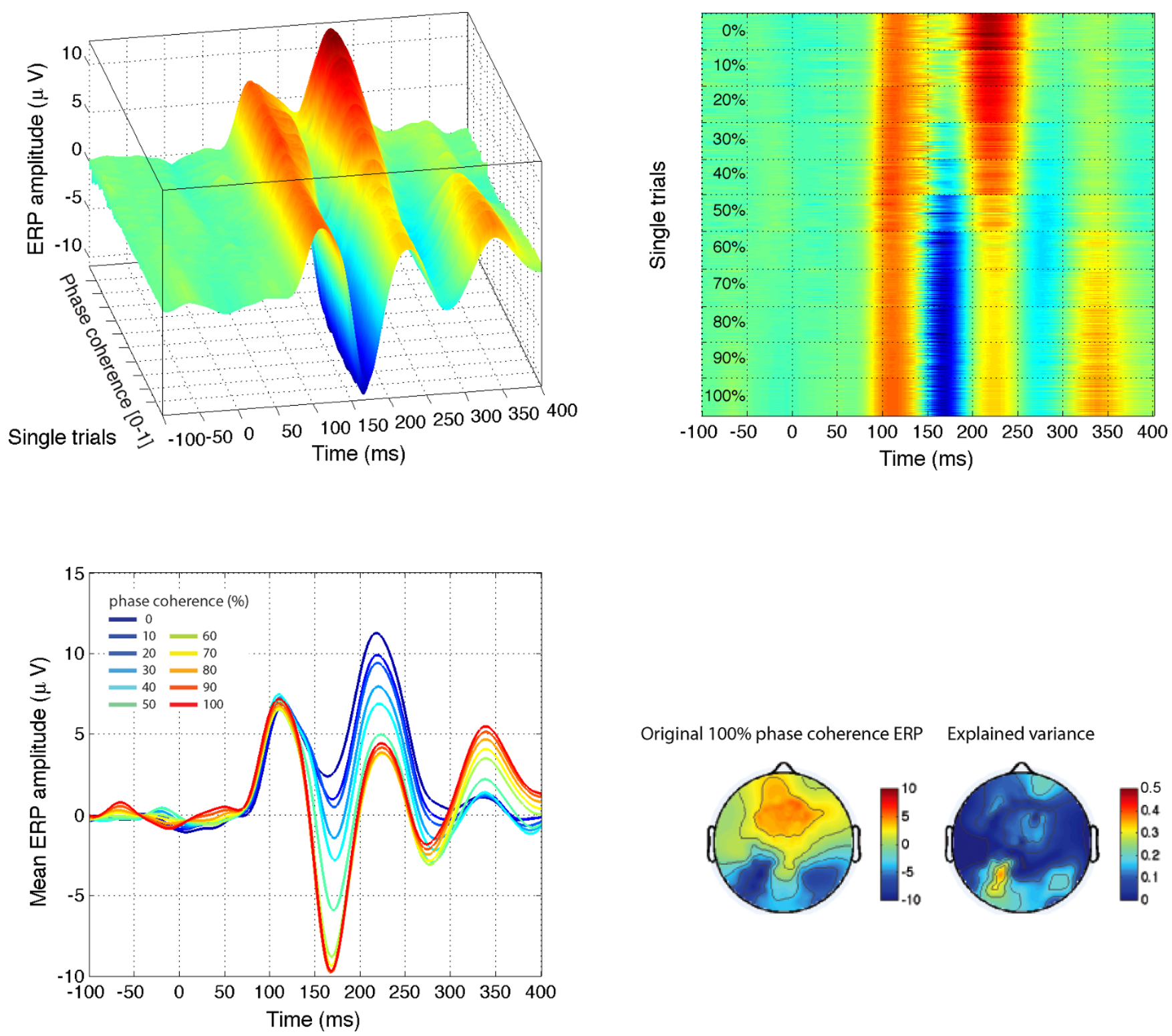

Figure 16

Modelled data and topographical maps from session I of subject RXL in Experiment 3. The topographic maps show data at the latency of maximum $R^{2}(168 \mathrm{~ms})$. This subject showed the earliest and the strongest sensitivity to phase noise. However, sensitivity to phase noise only starts to emerge at about $150 \mathrm{~ms}$ after stimulus onset, compared to about 120 ms in Experiment I. Figure caption details are otherwise identical to those of Figure 5.

stitute one way to explore face and object processing by creating an information space. This approach can be understood as an extension of classic categorical designs, in which regular stimuli are contrasted with noise (i.e. the two extreme points of the continuum). Finally, our approach can be applied to a large range of problems that researchers in behavioural neuroscience usually address using categorical designs.

\section{Authors' contributions}

GAR, PJB, ABS designed the study. GAR collected the data, conducted the analyses and wrote the manuscript. CRP helped analyse the data. CRP and PJB helped revise the manuscript. All authors read and approved the final manuscript.

\section{Acknowledgements}

NSERC Discovery Grants 42133 and 105494, and Canada Research Chairs supported PJB and ABS. A CIHR fellowship grant and a British Academy 
grant supported GAR. We thank Jesse Husk, Sandra Thomson, Melissa Sergi, and Richard Louka for their help collecting data.

\section{References}

I. Bullier J, Hupe JM, James AC, Girard P: The role of feedback connections in shaping the responses of visual cortical neurons. Prog Brain Res 2001, I34: 193-204.

2. Bullier J: Integrated model of visual processing. Brain Research Reviews 200I, 36(2-3):96-107.

3. DiCarlo JJ, Cox DD: Untangling invariant object recognition. Trends Cogn Sci 2007, I I (8):333-4I.

4. Rust NC, Movshon JA: In praise of artifice. Nat Neurosci 2005, 8(12): 1647-50.

5. Felsen G, Dan Y: A natural approach to studying vision. Nat Neurosci 2005, 8( I 2): 1643-6.

6. Logothetis NK, Sheinberg DL: Visual object recognition. Annu Rev Neurosci 1996, 19:577-621.

7. Grill-Spector K, Kushnir T, Hendler T, Edelman S, Itzchak Y, Malach $\mathrm{R}$ : A sequence of object-processing stages revealed by $\mathrm{fMR}$ in the human occipital lobe. Hum Brain Mapp 1998, 6(4):3 I6-28.

8. Tanaka K: Columns for complex visual object features in the inferotemporal cortex: clustering of cells with similar but slightly different stimulus selectivities. Cereb Cortex 2003, I3(I):90-9.

9. Tjan BS, Lestou V, Kourtzi Z: Uncertainty and invariance in the human visual cortex. J Neurophysiol 2006, 96(3): I 556-68.

10. Hoffman KL, Gothard KM, Schmid MC, Logothetis NK: FacialExpression and Gaze-Selective Responses in the Monkey Amygdala. Curr Biol 2007.

II. Pernet C, Schyns PG, Demonet JF: Specific, selective or preferential: comments on category specificity in neuroimaging. $\mathrm{Neu}$ roimage 2007, 35(3):991-7.

12. Thorpe S], Fabre-Thorpe M: Seeking categories in the brain. Science 200I, 29I(5502):260-263.

13. Rousselet GA, Thorpe SJ, Fabre-Thorpe M: How parallel is visua processing in the ventral pathway? Trends Cogn Sci 2004 8(8):363-370.

14. Schyns PG, Petro LS, Smith ML: Dynamics of visual information integration in the brain for categorizing facial expressions. Curr Biol 2007, I 7( I 8): I580-5.

15. Hillyard SA, Teder-Salejarvi WA, Munte TF: Temporal dynamics of early perceptual processing. Curr Opin Neurobiol 1998, 8(2):202-10.

16. Rossion B, Joyce CA, Cottrell GW, Tarr MJ: Early lateralization and orientation tuning for face, word, and object processing in the visual cortex. Neuroimage 2003, 20(3): 1609-24.

17. Itier RJ, Latinus M, Taylor MJ: Face, eye and object early process ing: what is the face specificity? Neuroimage 2006, 29(2):667-76.

18. Rousselet GA, Macé MJM, Fabre-Thorpe M: Animal and human faces in natural scenes: How specific to human faces is the NI 70 ERP component? J Vis 2004, 4(I): I3-2I.

19. Bentin S, Mouchetant-Rostaing Y, Giard MH, Echallier JF, Pernier J: ERP manifestations of processing printed words at different psycholinguistic levels: time course and scalp distribution. Cogn Neurosci 1999, I I (3):235-260.

20. Jeffreys DA, Tukmachi ES: The vertex-positive scalp potential evoked by faces and by objects. Exp Brain Res 1992 9 I(2):340-50

21. Jeffreys DA, Tukmachi ES, Rockley G: Evoked potential evidence for human brain mechanisms that respond to single, fixated faces. Exp Brain Res 1992, 9 I(2):35I-62

22. Bötzel K, Schulze S, Stodieck SR: Scalp topography and analysis of intracranial sources of face-evoked potentials. Exp Brain Res 1995, 104:135-43.

23. Joyce $C$, Rossion $B$ : The face-sensitive NI 70 and VPP components manifest the same brain processes: the effect of reference electrode site. Clin Neurophysiol 2005, I I 6( I I ):26 I 3-3 I

24. Bentin S, Taylor MJ, Rousselet GA, Itier RJ, Caldara R, Schyns PG, Jacques $C$, Rossion $B$ : Controlling interstimulus perceptual variance does not abolish N 70 face sensitivity. Nat Neurosci 2007 I 0(7):80I-2.

25. VanRullen R, Thorpe SJ: The time course of visual processing: from early perception to decision- making. J Cogn Neurosc 200I, I3(4):454-6I.
26. Itier RJ, Taylor MJ: NI70 or NI? Spatiotemporal differences between object and face processing using ERPs. Cereb Cortex 2004, I 4(2): 132-42.

27. Rousselet GA, Macé MIM, Thorpe SJ, Fabre-Thorpe M: Limits of ERP differences in tracking object processing speed. J Cogn Neurosci 2007, I9(8): |24|-58.

28. Rousselet GA, Husk JS, Bennett PJ, Sekuler AB: Time course and robustness of ERP object and face differences. Journal of Vision 2008, 8( ( 2):3.

29. Jemel B, Schuller AM, Cheref-Khan Y, Goffaux V, Crommelinck $M$, Bruyer R: Stepwise emergence of the face-sensitive NI70 event-related potential component. Neuroreport 2003 | 4( I 6):2035-9.

30. Philiastides MG, Ratcliff R, Sajda P: Neural representation of task difficulty and decision making during perceptual categorization: a timing diagram. J Neurosci 2006, 26(35):8965-75.

31. Philiastides MG, Sajda P: Temporal characterization of the neural correlates of perceptual decision making in the human brain. Cereb Cortex 2006, I 6(4):509-I8.

32. Tanskanen T, Nasanen R, Montez T, Paallysaho J, Hari R: Face recognition and cortical responses show similar sensitivity to noise spatial frequency. Cereb Cortex 2005, I5(5):526-34

33. Tanskanen $T$, Nasanen $\mathrm{R}$, Ojanpaa H, Hari R: Face recognition and cortical responses: effect of stimulus duration. Neuroimage 2007, 35(4): 1636-44.

34. Hauk O, Davis MH, Ford M, Pulvermuller F, Marslen-Wilson WD The time course of visual word recognition as revealed by linear regression analysis of ERP data. Neuroimage 2006 30(4): $1383-400$.

35. Schyns PG, Jentzsch I, Johnson M, Schweinberger SR, Gosselin F: A principled method for determining the functionality of brain responses. Neuroreport 2003, I4(13): I665-9.

36. Rousselet GA, Husk JS, Bennett PJ, Sekuler AB: Spatial scaling factors explain eccentricity effects on face ERPs. Journal of Vision 2005, 5(1 0): I.

37. Rousselet GA, Husk JS, Bennett PJ, Sekuler AB: Single-trial EEG dynamics of object and face visual processing. Neuroimage 2007, 36(3):843-62.

38. Morrone MC, Burr DC: Feature detection in human vision: a phase-dependent energy model. Proc $R$ Soc Lond B Biol Sci 1988 , 235( I 280):22 I-45

39. Thomson MG, Foster DH, Summers RJ: Human sensitivity to phase perturbations in natural images: a statistical framework. Perception 2000, 29:1057-1069.

40. Wichmann FA, Braun DI, Gegenfurtner KR. Phase noise and the classification of natural images. Vision Res 2006, 46(8-9): I 520-9.

4I. Drewes ], Wichmann FA, Gegenfurtner KR: Classification of natural scenes: Critical features revisited. Journal of Vision 2006, 6(6):561

42. Jacques $C$, Rossion B: Concurrent processing reveals competition between visual representations of faces. Neuroreport 2004, I5( I 5):24|7-2I.

43. Jacques $C$, Rossion $B$ : The time course of visual competition to the presentation of centrally fixated faces. J Vis 2006 6(2): $154-62$.

44. Loschky LC, Larson AM: Localized information is necessary for scene categorization, including the Natural/Man-made distinction. J Vis 2008, 8(I):4.

45. Joubert O, Rousselet GA, Fize D, Fabre-Thorpe M: Rapid visual categorization of natural scene contexts with equalized amplitude spectrum and increasing phase noise. Journal of Vision in press.

46. Dakin SC, Hess RF, Ledgeway T, Achtman RL: What causes nonmonotonic tuning of $\mathrm{AMRI}$ response to noisy images? Curr Biol 2002, I 2( I 4):R476-7. author reply $R 478$

47. DeCarlo LT: On the meaning and use of kurtosis. Psychol Meth 1997, 2(3):292-307.

48. Kingdom FA, Hayes A, Field DJ: Sensitivity to contrast histogram differences in synthetic wavelet-textures. Vision Res 2001, $4 I(5): 585-98$

49. Wilcox RR: Introduction to Robust Estimation and Hypothesis Testing. 2nd edition. Academic Press; 2005.

50. Thomson MG: Higher-order structure in natural images. J Opt Soc Am A 1999, 16(7): I549-I553.

51. Sekuler $A B$, Bennett PJ: Visual neuroscience: Resonating to natural images. Curr Biol 200 I, I I( I 8):R733-6. 
52. Olshausen BA, Field D): Sparse coding of sensory inputs. Curr Opin Neurobiol 2004, I 4(4):48I-7.

53. Gold J, Bennett PJ, Sekuler AB: Identification of band-pass filtered letters and faces by human and ideal observers. Vision Res 1999, 39(21):3537-60.

54. Husk JS, Bennett PJ, Sekuler AB: Inverting houses and textures: Investigating the characteristics of learned inversion effects. Vision Res 2007, 47(27):3350-9.

55. Oppenheim AV, Lim JS: The importance of phase in signals. Proceedings of the IEEE I98I, 69:529-54I.

56. Sekuler $A B$, Bennett PJ: Spatial phase differences can drive apparent motion. Percept Psychophys 1996, 58(2): 174-90.

57. Portilla J, Simoncelli EP: A parametric texture model based on joint statistics of complex wavelet coefficients. Int J Com Vis 2000, 40(I):49-7I.

58. Tucker DM: Spatial sampling of head electrical fields: the geodesic sensor net. Electroencephalogr Clin Neurophysiol 1993, 87(3): 154-63.

59. Delorme A, Makeig S: EEGLAB: an open source toolbox for analysis of single-trial EEG dynamics including independent component analysis. J Neurosci Methods 2004, 134(I):9-21.

60. Delorme A, Sejnowski T, Makeig S: Enhanced detection of artifacts in EEG data using higher-order statistics and independent component analysis. Neuroimage 2007, 34(4): 1443-9.

61. Simoncelli EP, Olshausen BA: Natural image statistics and neural representation. Annu Rev Neurosci 2001, 24: I 193-2I6.

62. Olman CA, Ugurbil K, Schrater P, Kersten D: BOLD fMRI and psychophysical measurements of contrast response to broadband images. Vision Res 2004, 44(7):669-83.

63. Rainer G, Augath M, Trinath T, Logothetis NK: Nonmonotonic noise tuning of BOLD $\mathrm{AMRI}$ signal to natural images in the visual cortex of the anesthetized monkey. Curr Biol 200I, I I:846-854.

64. Rainer G, Augath M, Trinath T, Logothetis NK: The effect of image scrambling on visual cortical BOLD activity in the anesthetized monkey. Neuroimage 2002, I6(3 Pt I):607-16.

65. Itier RJ, Taylor MJ: Source analysis of the NI70 to faces and objects. Neuroreport 2004, I 5(8): I 26I-5.

66. Itier RJ, Herdman AT, George N, Cheyne D, Taylor MJ: Inversion and contrast-reversal effects on face processing assessed by MEG. Brain Res 2006, III 5(I): 108-20.

67. Murray MM, Wylie GR, Higgins BA, Javitt DC, Schroeder CE, Foxe J]: The spatiotemporal dynamics of illusory contour processing: combined high-density electrical mapping, source analysis, and functional magnetic resonance imaging. J Neurosci 2002, 22(1 2):5055-73.

68. Sehatpour P, Molholm S, Schwartz TH, Mahoney JR, Mehta AD, Javitt DC, Stanton PK, Foxe JJ: A human intracranial study of longrange oscillatory coherence across a frontal-occipital-hippocampal brain network during visual object processing. Proc Natl Acad Sci USA 2008, 105(I I):4399-404.

69. Smith ML, Gosselin F, Schyns PG: From a face to its category via a few information processing states in the brain. Neuroimage 2007, 37:974-84. http.//www.biomedcentral.com/1471-2202/9/98 